\title{
Highly Stretchable, Directionally-oriented Carbon Nanotube/PDMS Conductive Films with Enhanced Sensitivity as Wearable Strain Sensors
}

\author{
Mehmet O. Tas a, Mark. A. Baker ${ }^{b}$, Mateus G. Masteghin a Jedidiah Bentz ${ }^{c}$, Keir Boxshall c, \\ Vlad Stolojan *, a \\ a Advanced Technology Institute, Department of Electronic Engineering, University of Surrey, Guildford, GU2 7XH, UK \\ ${ }^{b}$ Department of Mechanical Engineering Sciences, University of Surrey, Guildford, GU2 7XH, UK \\ c Smiths Interconnect, 8851 SW Old Kansas Ave., Stuart, Florida 34997, USA
}

\begin{abstract}
:
Recent interest in the fields of human motion monitoring, electronic skin and human-machine interface technology demand strain sensors with high stretchability/compressibility $(\varepsilon>50 \%$ ), high sensitivity (or gauge factor (GF > 100) and long-lasting electromechanical compliance. However, current metal and semiconductor-based strain sensors have very low $(\varepsilon<5 \%)$ stretchability or low sensitivity $(G F<2)$, typically sacrificing the stretchability for high-sensitivity. Composite elastomer sensors are a solution where the challenge is to improve the sensitivity to GF $>100$. We propose a simple, low cost fabrication of mechanically compliant, physically robust metallic carbon nanotube (CNT)-polydimethylsiloxane (PDMS) strain sensors. The process allows the alignment of CNTs within the PDMS elastomer, permitting directional sensing. Aligning CNTs horizontally (HA-CNTs) on the substrate before embedding in the PDMS reduces the number of CNT junctions and introduces scale-like features on the CNT film perpendicular to the tensile strain direction, resulting in improved sensitivity compared to vertically-aligned CNT-(VA-CNT)PDMS strain sensors under tension. The CNT alignment and the scale-like features modulate the electron conduction pathway, affecting the electrical sensitivity. Resulting GF are 594 at $15 \%$ and 65 at $50 \%$ strains for HA-CNT-PDMS and 326 at $25 \%$ and 52 at $50 \%$ strains for VA-CNT-PDMS sensors. Under compression, VA-CNT-PDMS show more sensitivity to small-scale deformation than HA-CNT-PDMS due to the CNT orientation and the continuous morphology of the film, demonstrating that the sensing ability can be improved by aligning the CNTs in certain directions. Furthermore, mechanical robustness and electromechanical durability are tested for over 6000 cycles to up to $50 \%$ tensile and compressive strains, with good frequency response with negligible hysteresis. Finally, both types of sensors are shown to detect small-scale human motions, successfully distinguishing various human motions with reaction and recovery times of as low as $130 \mathrm{~ms}$ and $0.5 \mathrm{~s}$ respectively.
\end{abstract}

KEYWORDS: Stretchable strain sensor, polydimethylsiloxane (PDMS), human motion monitoring, carbon nanotube, aligned-CNT composite sensors

*email address: v.stolojan@surrey.ac.uk, Tel: +44 (0)1483 689411 


\section{Introduction:}

In recent years, there has been a growing demand for stretchable and soft electronic devices ${ }^{1,2}$ for applications such as wearable health-monitoring devices ${ }^{3,4}$ and artificial skin. ${ }^{5,6}$ Stretchable strain sensors, in which the mechanical deformation is quantified through a corresponding change in the electrical response (capacitance or resistance), are used to detect human motion. However, applications such as pulse monitoring or respiration can demand high sensitivity or gauge factors (GF), as well as high stretchability for more sizeable human motion detection applications such as the bending of fingers, arms or legs, where mechanical strains up to $55 \%$ are generated due to the movement of joints. ${ }^{5}$ Current metal and semiconductor-based strain sensors have limited sensitivity (GF $<2)$ and stretchability $(\varepsilon<5 \%)$ due to the brittleness of the sensing materials. ${ }^{7}$ Continual epidermal electronics applications require a combination of good stretchability $(\varepsilon>50 \%$ ) with a high sensing ability or GF (GF $>100$ ), combined with high durability and with good electromechanical compliance for long-term. Nanoscale materials have been used extensively as building blocks within elastomer matrices to fabricate inventive strain sensors with enhanced performance. Recently, highly-sensitive graphene-based strain sensors have been reported ${ }^{8-10}$, but these sensors displayed low stretchability due to the brittleness of the graphene sheets. Yan et al. ${ }^{11}$ reported stretchability of up to $100 \%$ (double in length) from a graphene-nanocellulose nanopaper, however the GF was 7.1 and the authors did not comment on the elastic recovery of the stretched films and/or durability concerns. Similarly, nanoparticles ${ }^{12}$ and nanowire-elastomer hybrid structures ${ }^{13,14}$ have been studied as sensitive strain sensors, yet they showed poor adhesion ${ }^{15,16}$ to the flexible substrates and the stretchability with good electrical characteristics has been proven to be difficult to achieve ${ }^{16}$ Finally, liquid-state-based sensing materials (such as; liquid metal and ionic-liquid) elastomer strain sensors have also been reported showing good stretchability (up to $550 \%)^{17}$ with very low hysteresis ${ }^{18}$ however also very low GF values $(\mathrm{GF}<5)$. 
Carbon nanotubes (CNTs) have been used broadly as strain sensors, actuators and also in flexible electronics due to their exceptional mechanical and electronic properties. ${ }^{5,19}$ The concentric-graphene layered nature of CNTs permits internal conductive channels which can be disturbed easily when stretched and compressed, exhibiting a measurable electrical signal change. ${ }^{20}$ Lately, there have been several studies combining the CNTs with elastomers for strain and pressure sensing applications. ${ }^{7,21-31}$ Cohen et al. ${ }^{25}$ fabricated percolating CNT networks on silicone elastomer via laser etching and filtering with good stretchability (up to $100 \%$ ) but with low GF $(<0.99)$. Song et al. ${ }^{32}$ reported A GF value of 4739 via a CNT-to-CNT contact, fabricated on Si electrodes fixed on a Polydimethylsiloxane (PDMS) substrate, however the applied strains were below $23 \%$, limiting the application range; additionally, processes such as photolithography and reactive ion etching (RIE) were employed which are expensive cleanroom practices. Similar results are reported recently where studies show either good stretchability (> 50\%) or high sensitivity (GF $<50$ ) but not both at the same time. ${ }^{27,29,30,33}$ Zhou et al. ${ }^{22}$ achieved GFs up to $10^{7}$ at $50 \%$ strain, for a CNT-based ecoflex film via the fragmentation of the CNT film, but these films showed slow response times of the order of a few seconds restraining the potential applications for human motion detection. Very recently, Chen et al..$^{23}$ reported highly sensitive (of up to 1665.9 GF) CNT-Ecoflex films with very good stretchability (> $100 \%$ ) however, these films are engineered by strong acid etching (sulfuric acid) of the expensive ecoflex substrate and relied on the sandwiched structures which are not as mechanically robust as nanocomposites subjected to shear stresses. ${ }^{34,35}$

Hecht et al. ${ }^{36}$ showed that the conductivity of a CNT bundle highly depends on the bundle orientation and the individual CNT length. Firstly, they demonstrated that when CNTs are aligned in parallel to the electric current direction the conductivity was higher (up to two folds) due to having a fewer number of CNT-toCNT junctions compared to a randomly arranged CNT network. Additionally, increasing the CNT length to up to $30 \mu \mathrm{m}$ in the bundles increased the conductivity even further. In terms of highly stretchable sensors, achieving an aligned CNT structure will provide a more conductive nanocomposite film which then could 
increase the sensitivity. Pu et al. ${ }^{37}$ reported that $2 \mathrm{D}$ end-to-end conductive networks of multi-walled carbon nanotubes (MWCNTs) realized in a copolymer matrix resulted in 125 times higher GF values (GF up to 248 ) compared to randomly aligned networks at very small strains (5\%). Furthermore, Lee et al. ${ }^{35}$ very recently showed that overlapping CNTs on an elastomer (Ecoflex) could result it very high GF values $(G F=42300)$ at large strains $125-145 \%$ based on the separation of the overlapped CNTs, however these sensors had large electrical hysteresis. ${ }^{4}$ Finally, similar results are reported here for a carbon nanofiber(CNF)-PDMS based stretchable sensors. ${ }^{38}$ In this paper, we propose a simple, cost effective process to form CNT-PDMS hybrid films with directional CNT orientation within the PDMS matrix. This method allows retaining of the CNT orientation, horizontal or vertical, which enhances the film robustness and directional sensing abilities. Most of the previous CNT-based sensors discussed above are fabricated through expensive and complex processes, without the ability to control the CNT alignment. Additionally, CNTs are deposited on to flexible substrates via filtering, spraying or printing rather than forming a nanocomposite structure. ${ }^{23,27,29,30,33}$ We show that, with this method, the CNTs can be aligned in either vertical or horizontal directions within the PDMS matrix. This increases the durability and the robustness of the devices, whilst the directionality of the CNTs affects the sensing abilities due the difference in the electromechanical response under tensile and compressive strains. To our knowledge, this is the first study in which CNTs are aligned in both horizontal and vertical directions in an elastomer and tested for mechanical robustness and electromechanical performance. GF values of up to 594 at $15 \%$ and 65 at 50 $\%$ tensile strains are demonstrated, depending on the CNT orientation. To our knowledge, this is one of the highest combination of the stretchability-sensitivity values reported for nanomaterials-PDMS based strain sensors (see Figure S1 in supplementary information for comparison data). Moreover, we show that composite films have tensile stresses over $4 \mathrm{MPa}$ at $50 \%$ strains and show no mechanical or sensitivity degradation after over 6000 cycles with fast response (as low as $130 \mathrm{~ms}$ ) and good recovery times (between $0.5-2 \mathrm{~s}$ ). 
a)

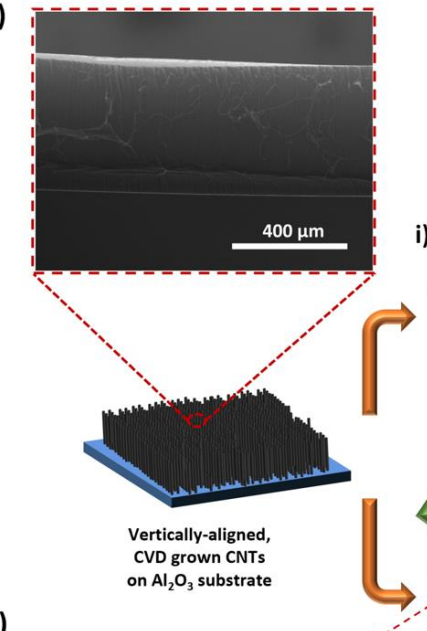

b)
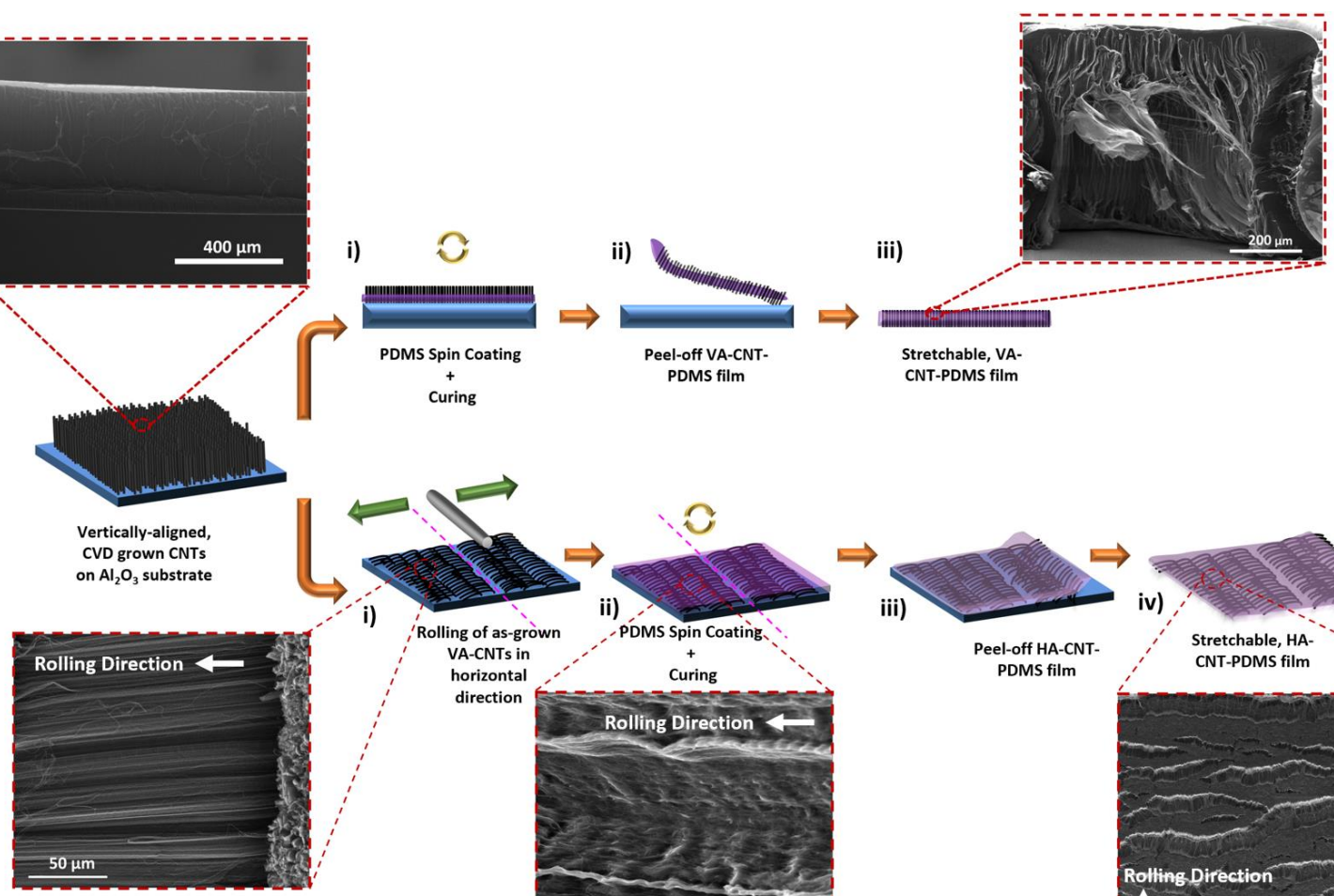

i)

uring

CNT-PDMS film

c)

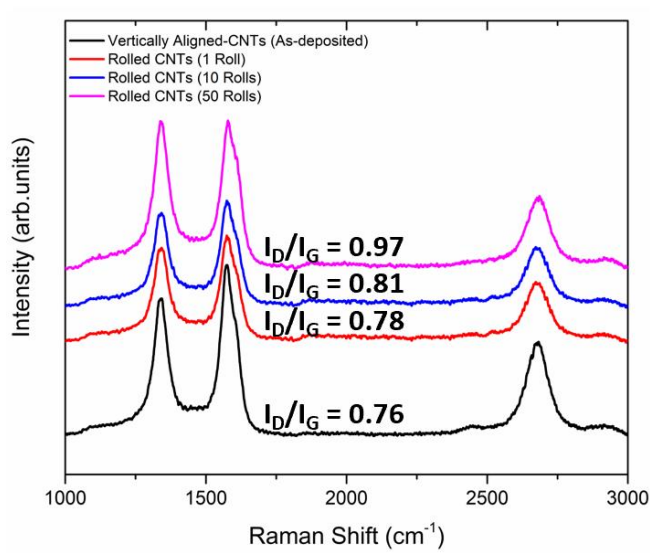

d)

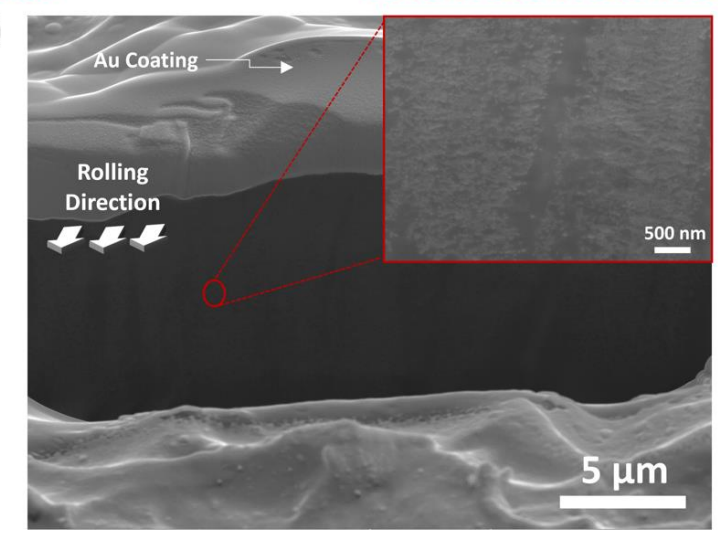

Figure 1. Fabrication of directionally oriented CNT-PDMS films: (a) The workflow for the VA-CNT-PDMS. VA-CNTs are deposited on $\mathrm{Al}_{2} \mathrm{O}_{3}$ substrates, followed by the spin coating of the PDMS (i). Then the nanocomposite film is peeled off using a blade (ii). (iii) Stretchable VA-CNT-PDMS strain sensor (b) HA-CNT-PDMS films are fabricated by rolling a hollow metal rod away from the centre of the substrate across an axis. Rolling process is repeated a few times to densify the CNT film in the horizontal direction. (ii) PDMS is then spin-coated before it is peeled off from the substrate (iii). (iv) is the final stretchable HA-CNT-PDMS film. Spin coating of the PDMS is performed in sequence to maintain either the vertical or the horizontal alignment. The sequence is as follows: 250 rpm for $30 \mathrm{~s}, 500 \mathrm{rpm}$ for $30 \mathrm{~s}, 1000 \mathrm{rpm}$ for $1 \mathrm{~min}, 3000 \mathrm{rpm}$ for $1 \mathrm{~min}$ and $4000 \mathrm{rpm}$ for $1 \mathrm{~min}$. CNT-PDMS composite films are cured at $85^{\circ} \mathrm{C}$ for one hour. (c) is the normalised Raman spectra of the CNT films before spin-coating the PDMS. The spectra show the characteristic Raman bands; a D peak at around $1340 \mathrm{~cm}^{-1}$, a G peak at around $1580 \mathrm{~cm}^{-1}$ and a distinct 2D peak at around $2690 \mathrm{~cm}^{-1}$. When the rolling process is repeated many times, a small increase in the $I_{D} / I_{G}$ ratio is observed which is an indication of induced structural defects. (d) is a focused ion beam (FIB) cross section of the HA-CNT-PDMS film showing the horizontal alignment, inset is the scanning electron microscopy (SEM) image of HA-CNTs pointing towards the rolling direction. 


\section{EXPERIMENTAL SECTION:}

\section{CNT Growth:}

A photo-thermal chemical vapour deposition (PT-CVD) method is used for CNT growth; the details of this system are described elsewhere. ${ }^{39-42}$ Briefly, it is a system where the optical heat is efficiently coupled with the CNT growth front, whilst the substrate is constantly water-cooled, resulting in a up to $300^{\circ} \mathrm{C}$ temperature gradient between the growth front and the substrate. CNT growth is carried out on $\mathrm{Al}_{2} \mathrm{O}_{3}$ substrates $(5 \mathrm{~cm} \times 5 \mathrm{~cm})$, where a $3 \mathrm{~nm}$ Fe-catalyst layer is evaporated onto a $10 \mathrm{~nm}$ of Al wetting layer. The PTCVD system is operated at $55 \%$ optical power which corresponds to $420^{\circ} \mathrm{C}$ substrate temperature measured via a pyrometer. The catalyst is annealed for $5 \mathrm{~min}$ under $100 \mathrm{sccm} \mathrm{H}_{2}$, followed by $10 \mathrm{~min}$

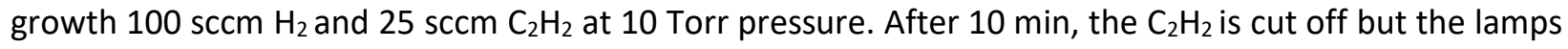
are kept on for an extra minute to avoid a layer of amorphous carbon building on the CNT forest. Asdeposited CNTs are vertically-aligned, multi-walled, metallic CNTs, with average length of $450 \mu \mathrm{m}$ (Figure 1a).

\section{Directionally-aligned CNT-PDMS sensor fabrication:}

PDMS is chosen as the elastomer due its high elasticity, good response to fast speed strains, inertness and non-toxic nature. ${ }^{4}$ it is also cheaper than Ecoflex. PDMS (Sylgard 184 Silicone Elastomer Kit, Dow Corning) is prepared by mixing the prepolymer and the curing agent with 10:1 ratio and degassed for 30 min under vacuum. Spin coating of the PDMS is performed in sequence to maintain either the vertical or the horizontal alignment. The sequence is as follows: $250 \mathrm{rpm}$ for $30 \mathrm{~s}, 500 \mathrm{rpm}$ for $30 \mathrm{~s}, 1000 \mathrm{rpm}$ for $1 \mathrm{~min}$, $3000 \mathrm{rpm}$ for $1 \mathrm{~min}$ and $4000 \mathrm{rpm}$ for $1 \mathrm{~min}$. CNT-PDMS composite films are cured at $85^{\circ} \mathrm{C}$ for one hour. VA-CNT-PDMS sensors are fabricated by spin coating (Figure 1a-i) PDMS on to an as-grown CNT forest on substrate, and then peeling of the film (Figure 1a-ii) from the substrate with the help of a blade. As-grown vertically-aligned CNTs on alumina substrates are rolled using an $8 \mathrm{~mm}$ diameter hollow metal rod (Figure 1-b-i) to achieve horizontal alignment of the CNTs. The metal rod is placed in the centre of the substrate 
and rolled towards the edge in opposite directions so that alignment would be along one axes and wouldn't be interrupted during spin coating due to PDMS's centrifugal force. The process is repeated a few times to achieve a dense continuous horizontal film. Then, the PDMS is spin coated (Figure 1-b-ii) and cured as described above, before the HA-CNT-PDMS nanocomposite film is peeled off from the substrate (Figure 1-b-iii). Finally, films are cut into $5 \mathrm{~mm} \times 20 \mathrm{~mm}$ rectangles. The final thicknesses of the films are $\sim 400 \mu \mathrm{m}$ for VA-CNT-PDMS and $\sim 200 \mu \mathrm{m}$ for HA-CNT-PDMS films.

\section{Electromechanical Characterisation:}

Electromechanical characterization of the composite films is carried out using a reconfigured setup described here ${ }^{43}$, consisting of a high-precision, ultra-low load, single-point load cell (Tedea-Huntleigh 0.3 kg with a load cell amplifier) attached to a motorised z-stage (Thorlabs $300 \mathrm{~mm}$ DC Servo Stage with TDC 001 controller, controlled via Kinesis $^{\complement}$ software), a displacement laser (Keyence LK-G) and a source/measure unit (see supplementary information Figure S2 for the experimental setup visualization). For tensile stress-strain tests, CNT-PDMS films are affixed between a base stage and a motorized stage via Cu clamps. Compressive stress-strain are performed with a $4.5 \mathrm{~mm}$ diameter flat, polished Cu top contact (again attached to the motorised stage). Force, displacement, electrical resistance and time are measured simultaneously using a Labview routine. Electrical measurements are performed by four-point resistance measurements using an Agilent B2902A. Electrical signal measurements used for human motion detection tests are performed by two-point resistance measurements by attaching two standard wires on the CNTPDMS films using silver paste. All electrical resistance signal measurements are performed at a constant voltage of $2.5 \mathrm{~V}$.

\section{Raman Characterisation:}

Raman Spectroscopy is employed to quantify the quality of the as-grown CNT columns, using a $532 \mathrm{~nm}$ laser wavelength, with a $1 \mu \mathrm{m}$ spot size and a 100x objective (Horiba XploRA Plus). The power density of the laser is kept below $70 \mu \mathrm{W} / \mu \mathrm{m}^{2}$ to prevent the heating-induced damaging of the sample and thermal 
shifting of the peaks. ${ }^{44}$ The scanned range is from 50 to $3500 \mathrm{~cm}^{-1}$, to include all the first and second order resonance typical Raman features of CNTs. For each sample, 5 spectra are collected, and $I_{D} / I_{G}$ ratios are averaged.

\section{RESULTS and DISCUSSION:}

Figure $1(a-b)$ schematically depicts the fabrication process of the directionally-aligned CNT-PDMS hybrid strain sensors. The alignments of the CNTs in the vertical (Figure 1a) and the horizontal (Figure 1b) directions respectively are retained after nanocomposite strain sensors are fabricated. In both cases, PDMS causes the CNT films to densify due to capillary forces. In HA-CNT-PDMS films, the CNTs fold as bundles, forming a scale-like structure when rolled, as shown Figure 1(b-iv); it indicates that there is a surface region at the top of the as-grown CNT films which behaves as a fused region. The overall electrical and mechanical performance of the nanotubes is linked to their quality, which can be assessed using Raman spectroscopy. ${ }^{45-47}$ The D-peak of graphitic carbon is not normally active except in the presence of a defect ${ }^{48}$, therefore, its' relative height is proportional to the number of defects present in the sample. The ratios of the $D / G\left(I_{D} / I_{G}\right)$ band intensity ratio in the Raman spectra is widely used as an indication of sample quality for carbon-based materials. ${ }^{49,50}$ The quality of as-deposited CNTs before and after rolling is evaluated, shown in Figure 1 (c). The as-grown CNTs had an $I_{D} / I_{G}$ ratio of 0.76 (spectrum collected from the top of the CNT forest), whilst after flattening is increased to 0.97 . Subsequent number of rolls did not change this ratio outside the standard deviation. Whilst defects may have been introduced during the bending and stretching of the CNTs, it is also possible that the top of the CNT forest may have a higher intrinsic quality than the bulk of the CNT forest due to the way the growth process is terminated. ${ }^{43}$ It should be noted here that, when the CNTs are grown on Si wafers, the rolling method does not work due to lack of adhesion between the Si substrate and the CNT film resulting in sections of the CNT films detaching from the substrate. The $\mathrm{Al}_{2} \mathrm{O}_{3}$ substrate is much rougher than a standard polished Si substrate 
(see supplementary information Figure S3 for the substrate roughness analysis), which could have provided the better adhesion between the CNT film and the substrate. Figure 1(c) is a focused ion beam (FIB) cross section of the horizontally aligned-CNT (HA-CNT) PDMS film. A gold coating is evaporated on the film to prevent electron charging. We observe that the horizontal alignment is retained after the PDMS is spin-coated on the substrate, as demonstrated in the inset image, the CNTs are pointing towards the rolling direction, confirming the effectiveness of the rolling process.

a)

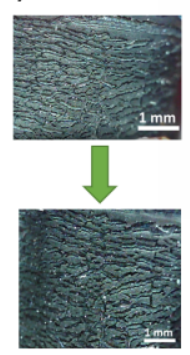

HA-CNT-PDMS under tension

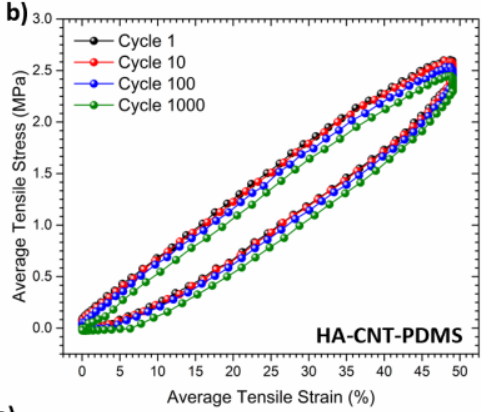

e)

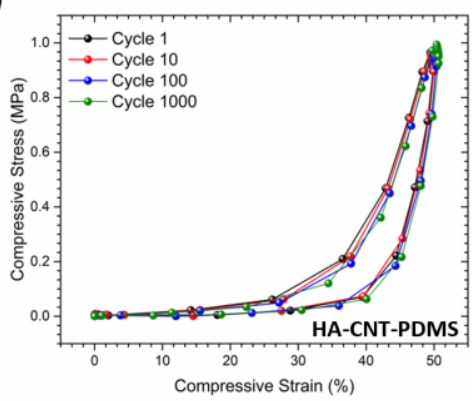

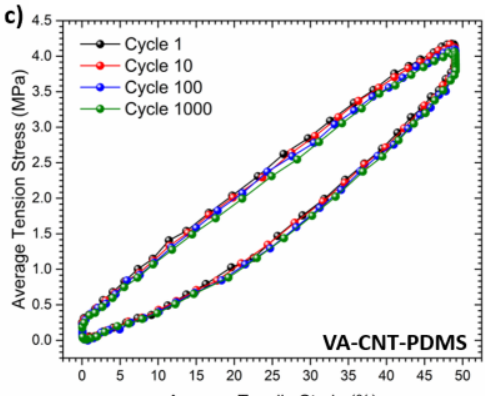

f)

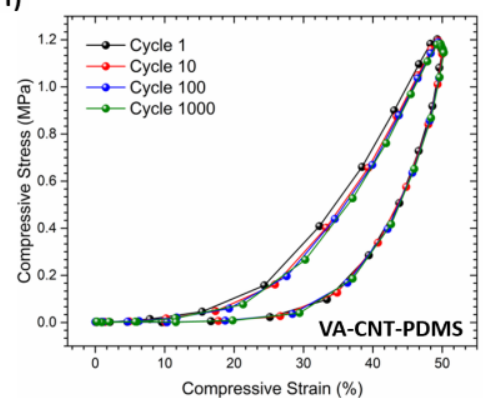

d)

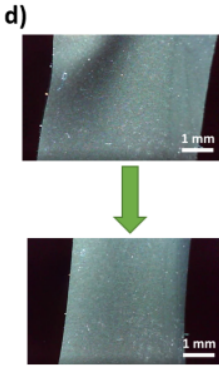

VA-CNT-PDMS

under tension g)

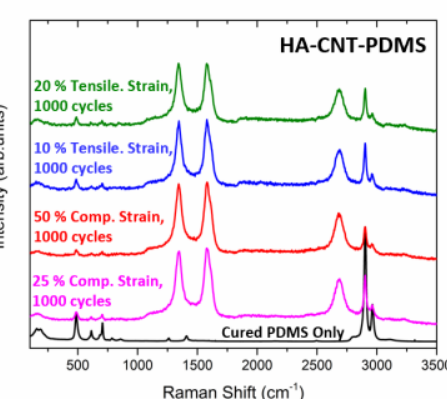

h)

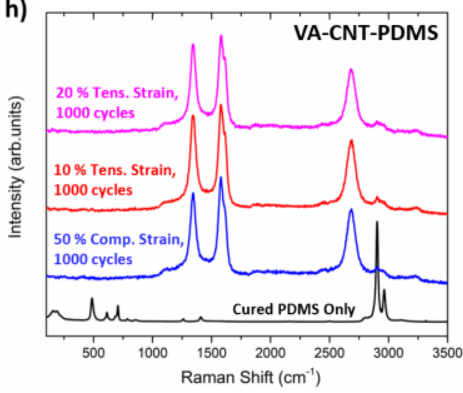

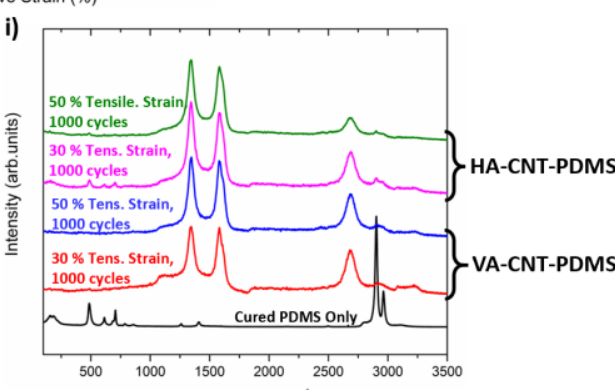

Figure 2. Mechanical characterization of the CNT-PDMS films under tensile and compressive strains. (a) is an in-situ optical image of HA-CNT-PDMS film under tension showing the extension of the scale-like structure. HA-CNT-PDMS film under cyclic (b) tensile and (e) compressive strains. (d) is an optical in-situ image of the VA-CNT sample under tension. VA-CNT-PDMS film under cyclic (c) tensile and (f) compressive strains. (g-i) Visible Raman spectra of the composite films under different cyclic loading conditions, showing the effect of cycling loading on the deformation of the CNT fibers within the PDMS matrix. Under high compressive strains up to $50 \%$ and low tensile strains $(<20 \%)$; c both HA-CNT-PDMS (g) and VA-CNT-PDMS (h) sensors' $I_{D} / I_{G}$ ratios remain within the standard deviation suggesting that no damage is introduced to the CNT fibers. However, when the tensile strains increased up to $50 \%, I_{D} / I_{G}$ ratios increased for both type with HA-CNT-PDMS (1.15 \pm 0.04$)$ showing larger ratios compared to VACNT-PDMS sensors $(1.07 \pm 0.04)$. The Raman data is collected from the tip of the CNTs for comparison, the measurements are 
repeated 5 times for each condition and averaged. The $I_{D} / I_{G}$ ratios are calculated by fitting Lorentzian functions to calculate the positions and the intensities of the corresponding peaks.

Figure 2 illustrates the mechanical evaluation of the fabricated strain sensors for human motion monitoring applications that demand mechanical durability and compliance under high dynamic strains. ${ }^{1,4,5,51}$ Firstly, Figure $2(\mathrm{a})$ and (d) are the in-situ images of the HA-CNT-PDMS and VA-CNT-PDMS sensors captured when subjected to tensile stresses. The morphologies of the films are considerably different, due to the horizontal alignment as depicted above in Figure $1(a, b)$, we observe scale-like features that are present due to the rolling of the CNTs. Under tension, these scales expand (Figure 2(a)), whereas VA-CNT-PDMS sensors exhibit a more uniform and continuous surface (Figure 2(d)). Figure 2(b) and (c) show the cyclic tensile loading of the films at $50 \%$ strain with a strain rate of $5 \mathrm{~mm} \mathrm{~s}^{-1}$. Firstly, we notice that more force is required ( $>4 \mathrm{MPa}$ ) to stretch the VA-CNT-PDMS sensors compared to HA-CNTPDMS ( 2.5 MPa), which can be explained by the apparent morphology of the films described above. Kim et al. ${ }^{52}$ reported tensile strengths up to $8 \pm 2 \mathrm{MPa}$ of $1 \mathrm{wt} . \%$ CNT-PDMS nanocomposite films and showed that the tensile strength decreased with increasing CNT concentration; however, better dispersion and CNT alignment provided higher strength. Similarly, the aligned CNTs in our study appear to provide better load transfer to the filler materials under high strains. After 1000 cycles, the loading-unloading curves show that the films do not plastically stretch. HA-CNT-PDMS sensors showed a slight decay $(<2 \%)$ in tensile strength as indicated by the 1000th cycle curve slightly shifting to a lower stress value; this could be explained by the slipping out of the suspended CNT-PDMS fibers across the scales, as discussed in the following section, as well as possible propagation of cracks/separation between the scales due to high strains.

It is also important to evaluate the performance under compressive stresses to understand the contact pressure limits of the sensors. In potential applications, these films are under multidirectional tensile and compressive stresses. ${ }^{29}$ Figure 2 (e-f) shows the mechanical behaviour of the sensors under cyclic compression forces, at $50 \%$ strains. Like in the case of the tensile stresses, the elasticity of the films is 
stable, corresponding to pressures of up to $1 \mathrm{MPa}$. For both type of sensors, the loading and unloading curves showed similar results, with VA-CNT sensors showing more mechanical resistance to applied pressure. When subjected to $25 \%$ strain, HA-CNT films showed less than $48 \mathrm{KPa}$ compression stress compared to $113 \mathrm{KPa}$ of VA-CNT-PDMS films. This could be attributed to the more homogeneous and continuous surface of the VA-CNT films in which the load-bearing ratio is higher. ${ }^{53}$ However, as the strain is increased, contact stresses increase exponentially resulting in two compression stages; first stage (up to $20-25 \%$ strain, low Young's Modulus < $200 \mathrm{KPa}$ ) and stage two, above $25 \%$ where the effective Young's modulus reaches up to 1.2 MPa. A cured, non-porous PDMS can have Young's Moduli between 0.4-3.5 $\mathrm{MPa}^{7}$; we believe the difference between the PDMS only film and the aligned CNT-PDMS nanocomposite films is because of the porous structure, as shown in Figure $1 \mathrm{a}$ and $\mathrm{b}$ ). This is due to the densification effect of the PDMS polymer based on the capillary forces acting upon the CNT forest as discussed in the following sections. When the films are compressed, the air is squeezed out of the empty spaces within the film. Chen et al. ${ }^{54}$ similarly showed the effect of porosity for randomly aligned CNT-PDMS nanocomposite films. Finally, the Young's modulus of human skin varies between $25-220 \mathrm{KPa}^{4}$, therefore the porosity in these films combined with the mechanical compliance can help imitate the elasticity of an actual human skin.

Figure 2(g-i) show the Raman spectra of the films after cyclic tension and compression loading. A cured, PDMS-only film Raman spectrum is included as reference. Because the strain sensing ability of the hybrid films depends on the CNTs structural stability, it is crucial to characterize the CNTs within the PDMS matrix after subjected to large strains under both tensile and compressive stresses. CNTs are proven to have excellent tensile strength ${ }^{55}$ however, under high strains aligned CNTs can slip out or deform, resulting in a failure of the CNT-PDMS interface. We note that $I_{D} / I_{G}$ ratios for HA-CNT-PDMS films showed a slight increase after testing at different conditions, as shown in Figure 2(g), when the tensile strain is increased from $10 \%$ to $20 \%, I_{D} / I_{G}$ ratio increased by $0.07 \pm 0.05$. Similarly, doubling the compressive strain from 25 
$\%$ to $50 \%$ resulted in $0.12 \pm 0.05$ increase in the ratio after testing for 1000 cycles. VA-CNT-PDMS sensors on the other hand, showed no change in the $I_{D} / I_{G}$ ratios (Figure $2 \mathrm{~h}$ ) under small tensile $(<30 \%)$ and large (up to $50 \%$ ) compressive strains. The intensity of the $D$ band increased with respect to $G$ band when nanocomposite sensors were tested at larger $30-50 \%$ tensile strains (Figure $2 \mathrm{~h}$ ) for both types of samples, however the HA-CNT-PDMS $I_{D} / I_{G}$ ratio is $(1.15 \pm 0.04)$ higher compared to VA-CNTS $\left(I_{D} / I_{G}=1.07 \pm\right.$ 0.03) tested under the same conditions. We attribute this to the breaking of the suspended CNT-PDMS fiber over the cracks as discussed above. It should be noted here that the characteristic PDMS-only peaks are more intense for the HA-CNT samples, which may indicate that, at the surface of the films, there is more PDMS compared to VA-CNTs; this confirms that the HA-CNTs are encapsulated more after the spin coating of the PDMS due to the rolling preparation. 


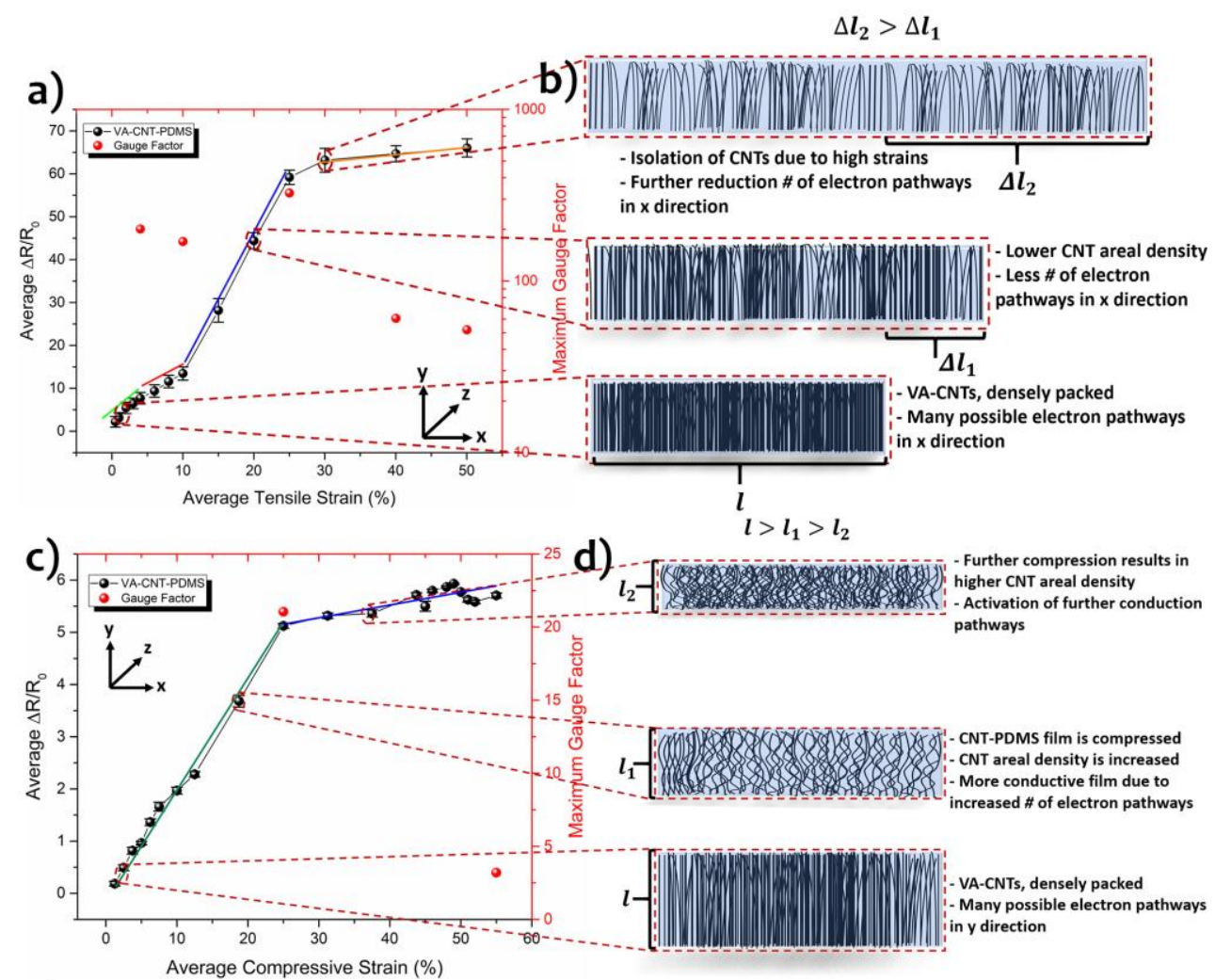

e)

Average Compressive Strain (\%)
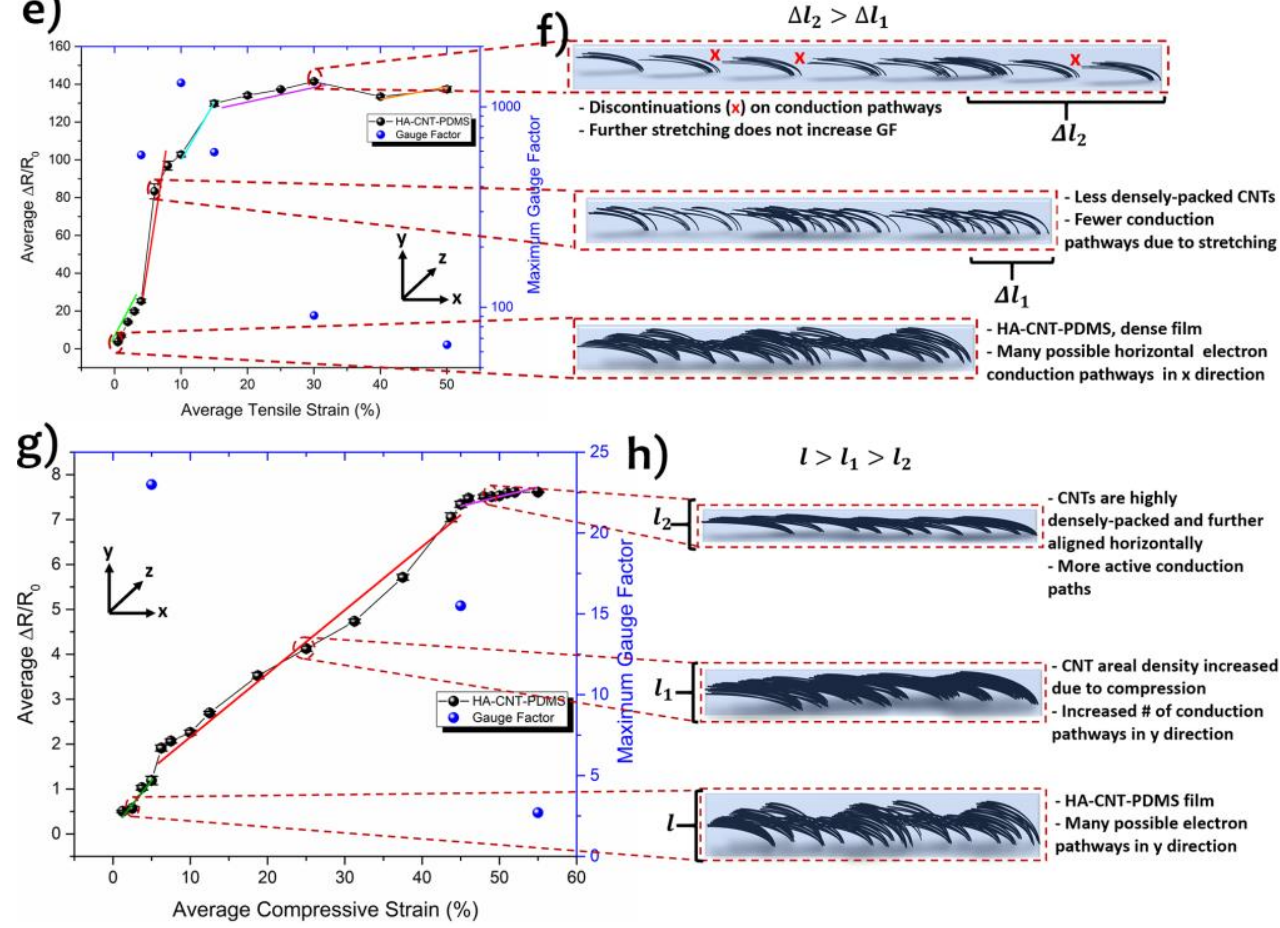

Figure 3. Electromechanical sensitivity of the aligned-CNT-PDMS sensors under (a)VA-CNT-PDMS sensor under tensile strains up to $50 \%$ with calculated maximum GF values at different strains, (b) corresponding conductivity mechanism. The model based on the temporary densification-separation of the aligned CNT forests within the PDMS matrix. When and (c) VA-CNT-PDMS sensor under compressive strains up to $55 \%$ with calculated maximum GF values at different strains. Under compression the films become more conductive, the GF values are reported as absolute values. (d) Working mechanism when films are under compression showing the temporary densification effect closing packing the CNTs and when compressed, the films become more 
conductive therefore the GF values reported are absolute values. (e) HA-CNT-PDMS sensors electromechanical sensitivity response under tensile strains up to $50 \%$ with calculated maximum GF values at different strains. (f) the working mechanism under tension, showing the separation of the CNTs and as well as opening of the CNT scale-like features, decreasing the number of conduction pathway. (g) HA-CNT-PDMS sensors electromechanical sensitivity response under compressive strains up to $55 \%$ with calculated absolute maximum GF values at different strains. (h) The working mechanism of HA-CNT-PDMS sensors under compressive strains showing the increased alignment, temporary densification and closing of the scale-like features increasing the conduction pathways resulting in a reduction in overall resistance of the sensor.

Figure 3 shows the electromechanical sensitivity of the sensors fabricated, under both tensile and compressive strains, together with proposed conductivity model. The lowest film resistance measured for HA-CNT-PDMS is $34.8 \Omega\left(2.18 \Omega / \mathrm{mm}^{2}\right)$ and $51.9 \Omega\left(3.24 \Omega / \mathrm{mm}^{2}\right)$ for VA-CNT-PDMS films under no strains (see supplementary information Figure S4). Both types of sensors are tested to up to $50 \%$ strains. Under tension, VA-CNT-PDMS sensors yield GF values over 326 at $25 \%$ and 52 at $50 \%$ strain respectively. (Figure 3a, see supplementary information for the GF calculation details). Initially, CNTs are densely-packed and vertically aligned in the PDMS matrix, allowing for many possible electron pathways. When stretched by a length of $\Delta l_{1}$ (Figure $3 \mathrm{~b}$ ), the CNTs start to separate from each other, resulting in a lower areal density; this then results in fewer conduction pathways along the stretching direction. Further stretching of the film by $\Delta l_{2}$ causes misalignment and isolation of the CNTs due to high strains, resulting in insulating regions and fewer still electron conduction pathways (see Figure S5 to see the cross-section SEM images of the sensor at different applied strains). Here it should be noted that, when materials are stretched, they usually contract in transverse direction based on the Poisson's ratio ( $v)$. This geometric effect is more pronounced in capacitive-type sensors since, when stretched, capacitive-type sensors undergo a change of capacitive area and a decrease in the thickness of the dielectric layer, resulting in an increase of capacitance $^{13}$ but usually both stretchable electrodes and dielectric layer assume to have the same Poisson's ratio, which means that when the applied strain is too large, the capacitive sensors start to deviate from linear sensitivity response. ${ }^{4}$ In CNT-based resistive-type of sensors, the change in the resistance with respect to applied strain originates from mechanisms such as disconnection of the sensing nanomaterial. The decrease in the cross-sectional area upon stretching could increase the CNT areal density however, the main working mechanism is the disconnections of the sensing CNT networks. $7,22,23,30$ 
When stretched, the cross-sectional area will decrease which means that the CNT density per area will increase increasing conduction, however the disconnections of the CNT network due to applied strain is the main working mechanism.

When subjected to increasing compressive strains, the absolute GF values decrease from 21 (at $25 \%$ ) to 3.2 at $55 \%$ applied strains (Figure 3c). When compressed from a film thickness of $l$ to $l_{1}\left(l>l_{1}\right)$, the sensor becomes more conductive (Figure 3d) due to the temporary densification of the CNTs and the closing of the insulating cracks, increasing the number of conduction pathways..$^{21,54}$ Further compression to $l_{2}$ $\left(l>l_{1}>l_{2}\right)$ increases the conductivity further but up to a limit, indicating that strain-induced CNT densification has reached saturation, and/or the film deforms laterally (rather than compress just in the direction of the applied force), such that the densification is reduced. Hu et al. ${ }^{21}$ also observed this limit using randomly-aligned CNT-PDMS nanocomposites and showed similar results.

HA-CNT-PDMS sensors under tensile strains (Figure 3e) show higher GF values of 594 at low strains (< $20 \%$ ) and up to 65 at $50 \%$ tensile strains, respectively. This can be attributed to the increased alignment of the CNTs within the matrix along the strain direction (see Figure S6 for the SEM images of the HA-CNTPDMS sensor subjected to different uniaxial tensile strains). Hecht et al. ${ }^{36}$ showed that, when CNTs are aligned, there are fewer CNT-to-CNT junctions compared to randomly distributed CNTs, decreasing the overall film resistance of the sensor (see supplementary information, Figure S4). Figure $3 f$ illustrates the proposed conduction mechanism for the HA-CNT-PDMS sensor when the film is stretched. Initially, the unstretched sensor of length $l$ has horizontally aligned, densely-packed CNT scales, as shown in the figure. When stretched by a length of $\Delta l_{1}$, CNTs start to separate from each other, resulting in a steep increase in the GF due to the reduction in the number of conduction pathways, as well as due to a rise in the number of CNT junctions. Increasing the strain further $\left(\Delta l_{2}\right)$ causes scales to expand and introduces discontinuous sections within the CNT film because of the lower CNT areal density. Once the number of conduction pathways is reduced, the resistance increases however at a lower rate, and the GF value 
settles. Further stretching does not increase the GF since further stretching already insulating sections does not change the sensitivity response. This model resembles the crack-induced electron transfer model for stretchable sensors. ${ }^{22,23}$ Zhou et al. ${ }^{22}$ achieved this by fragmentation of CNTs on PDMS and similarly Chen et al. ${ }^{23}$ used an acid solution to introduce cracks on the Ecoflex substrate, allowing crack-guided electron transfer. Other groups used lasers to introduce crack patterns. ${ }^{56,57}$ Our method does not require such further processes. Finally, Figure $3 g$ displays the behaviour of the HA-CNT-PDMS film under compressive strains. The sensor response is more linear compared to its behaviour under tension with, absolute GF values varying between 13 and 30, expect at very low strains (1.25\%), the GF value was 40 . One of the important aspects of a good strain sensor is the linear electromechanical response. $29,56,58$ Similar to the VA-CNT-PDMS sensors, when compressed, the composite film becomes more conductive due to the increased CNT areal density an closing-up of the scales as illustrated in Figure 3h. Compressive forces activate more conduction pathways, showing the importance of CNT alignment.

Directionally-aligned CNT-PDMS films are then analysed using scanning electron microscopy (SEM) under tensile and compressive strains as shown in Figure 4. For wearable strain sensing applications; sensors are subjected to dynamic multidirectional strains, as visualized in Figure 4a. The blue arrows and the color indicate tensile strains, whereas red arrows and color represent compressive strains. Figure $4 b-d$ show the HA-CNT-PDMS sensor under applied tensile strains, causing the scale-like features to enlarge and CNTs to stretch (Figure 4c) resulting in the formation of large gaps and disconnections within the film. This induces an increase in the film resistance, supporting the mechanism discussed in Figure 3 . Figure $4 d$ displays suspended CNT-PDMS fibers across a stretched across a crack, under mechanical deformation, we believe the stability of these fibers is easily disturbed further increasing the sensitivity. Throughout the film, the gap length varies from 5-200 $\mu \mathrm{m}$. Additionally, as can be seen in Figure S7 that, the PDMS elastomer also densifies the tubes allowing packing of CNTs more densely. Densification is a well-known practice to improve electrical and mechanical properties of CNTs. ${ }^{59,60}$ Furthermore, the buckling effect is 
observed (see Figure S7c), which we believe provides the extra mechanical compliance/sensitivity when sensors are subjected to strains. Creating buckled structures for initially rigid materials has been a widely used strategy in flexible sensors. ${ }^{11,57,61}$ When the film is compressed (Figure 4e-g) the widened gaps close up, decreasing the number of disconnections in the composite film by temporarily increasing the CNT areal density which then decreases the film resistivity. Figure $4 \mathrm{c}$ and Figure $4 \mathrm{f}$ has the same field of view and exhibits a good example of sensing, based on the separation and contraction of the HA-CNTs mechanism discussed in Figure 3. Compression and tension sides of the VA-CNT-PDMS are shown in Figure 4h. When stretched and wrapped around an object, it is possible to observe the separation of VA-CNTs in the polymer matrix. The colour graded triangles represent the separation of the CNTs on the tension side which closes up towards the compression side due to compressive strains. This supports the VA-CNTPDMS sensing mechanism described in Figure 3. Furthermore, Figure $4 \mathrm{k}$ and $\mathrm{I}$ show the uniaxially stretched films at $10 \%$ and $30 \%$ strains revealing the widening of gaps as the tensile stretching is increased. 

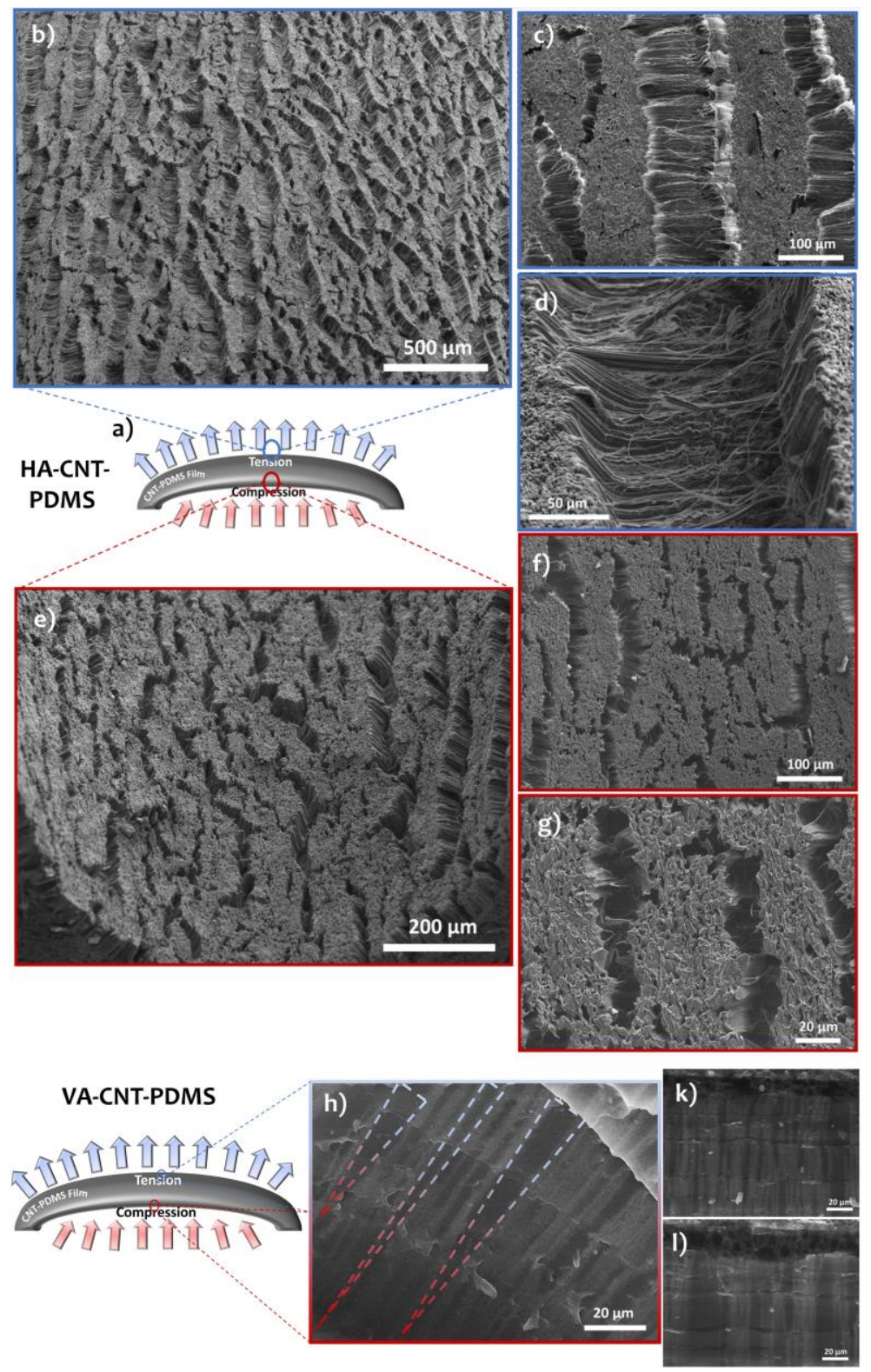

Figure 4. SEM images of the stretched and compressed sensors. (a) a visualisation of dynamic strains acting upon the sensors showing the tension and compression sides. (b-g) The HA-CNT-PDMS sensor under tensile and compressive strains. (b) A tilted image of the stretched film showing the enlargement of the scale-like features and increase in the disconnections in the film. (c) A top view of the sensor film. (d) A tilted image of a widen CNT-PDMS valley like feature with suspended CNTs across. (e-g) VACNT-PDMS film under compression. (e) Scale-like features are closed up due to applied compressive strains (f) A close up top view of the sensor film with the same view-field as (c) showing the difference in the disconnection width. $(\mathrm{g})$ a zoomed-in image of the closed-up scales and increase in the CNT areal density which result in increase in the conductivity. (h-I) The VA-CNT-PDMS film under tensile and compressive strains. (h) an SEM image of the cross-section of the film wrapped around a round object, showing the tension (blue region) and compressive sides (red region) of the film and the arrangement of the CNTs under strain where the tension increases the gaps between CNTs whereas compression temporarily increases the CNT areal density. (k) A cross-section of the uniaxially stretched film (10\%) showing the separation of the CNTs within the matrix. (I) a further stretched film at $30 \%$ strain. All tilt angles are $55^{\circ}$.

In addition to the high sensitivity and stretchability demonstrated in Figure 3, a human motion detection strain sensors should also be mechanically durable, with a good frequency response and low electrical 
hysteresis under mechanical deformation, since high, long duration and frequent strains may be applied during such applications. ${ }^{4}$ Figure 5 shows the electromechanical stability (Figure $5 a, d$ ), electrical signal response (Figure $5 b, e$ ) and electrical hysteresis (Figure $5 c, f$ ) for both types of sensor. Figure 5 shows the electromechanical stability of the HA-CNT-PDMS sensor under $50 \%$ tensile strain for over 6000 loadingunloading cycles with green lines showing the trace of the stability. The performance degradation after 6000 cycles remains below $5 \%$. VA-CNT-PDMS sensors (Figure $5 \mathrm{~d}$ ) shows a more stable electromechanical signal, with less than $2 \%$ stability degradation under $50 \%$ tensile strain. Both types of sensor display good stability, with small deviations from the average values. CNT-based composite strain sensors ${ }^{5,28}$ show better durability compared to other nanomaterial-based composites, such as nanowires. ${ }^{62}$ The high durability of CNT-based sensors is probably due to the high elastic behavior of CNTs, preventing plastic deformation and fracture of CNTs under dynamic loading. ${ }^{4,43}$ Figure 5 (b) and (e) show the electrical signal response to different frequencies. Frequency response time determines how quickly the strain sensors reach towards steady state response. Certain human motions, such as pulse monitoring or respiration, or motions like knee-jerk reflex can demand good electrical signal response at higher frequencies (up to 1 $\mathrm{Hz}) .{ }^{4,63}$ A $90 \%$ time constant $\left(\tau_{90 \%}\right)$ is generally accepted as the standard response time value for stretchable sensors. ${ }^{24}$ CNT-PDMS strain sensors are tested for up to $1 \mathrm{~Hz}$ and are capable of detecting mechanical deformation induced strain changes at high and lower frequencies.

Under dynamic loading, such as would occur with skin-attachable and wearable applications, electrical hysteresis behaviour becomes important. Large hysteresis results in irreversible sensing performance when strain sensors are subjected to dynamic loading. ${ }^{64}$ Figure 5 (c) and (f) show the electrical hysteresis for HA-CNT-PDMS and VA-CNT-PDMS sensors respectively, at tensile strains of $15 \%, 30 \%$ and $60 \%$. We observe that the hysteresis become visible with increasing applied strains. Hysteresis mainly originates form the elastomer and the interaction between the nanofiller material (in this case, CNTs) and the polymer. ${ }^{4,24,65}$ Amjadi et al. ${ }^{4}$ reported that increasing the interfacial binding between the filler and the 
elastomer results in lower hysteresis. When the binding is weak, the nanomaterials can slide into the elastomer matrix upon large tensile strains. Once released, they cannot rapidly slide back to their original positions, leading to high hysteresis. ${ }^{4,52,66}$ As discussed previously in Figure 2, the mechanical loadingunloading curves suggest that the interfacial binding between the aligned CNTs and the PDMS elastomer is strong even with a high strain rate $\left(10 \mathrm{~mm} \mathrm{~s}^{-1}\right)$, and this correlates with the low hysteresis observed for both type of sensors. The more pronounced hysteresis experienced at $60 \%$ strain for the HA-CNT-PDMS sensors could be the result of the buckling and sliding of the CNTs during loading and unloading, since for HA-CNTs the stretching direction is parallel to the CNT alignment direction whereas forces acting upon the VA-CNT sensors are in radial direction.
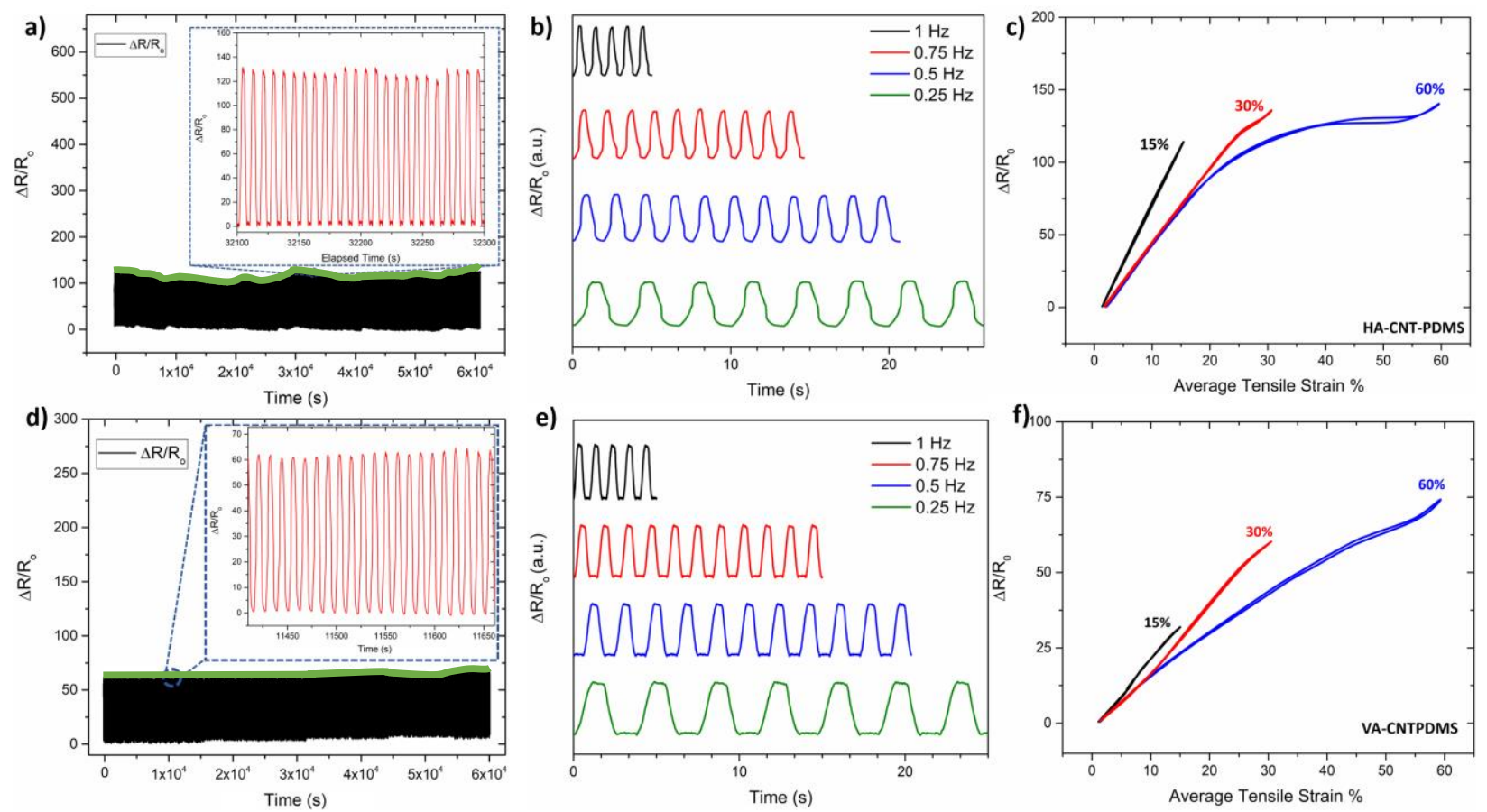

Figure 5. Electromechanical characteristics of the directionally oriented CNT-PDMS sensors. (a) Electromechanical durability after stretching the sensors for over 6000 times under cycling loading at $50 \%$ strain showing the stability of the electrical signal response for the HA-CNT-PDMS sensors with less than $5 \%$ degradation. Green line represents the electromechanical stability. (b) the electrical signal frequency response of the HA-CNT-PDMS film at $50 \%$ strain from $0.25 \mathrm{~Hz}$ to up to $1 \mathrm{~Hz}$ with good identical response, (c)Electrical hysteresis of the HA-CNT-PDMS sensor at different strain rates, no hysteresis is observed when tested up to $30 \%$ strain, when the sensors are stretched $60 \%$ the hysteresis starts to be visible. (d) Electromechanical durability of the VACNT-PDMS sensor after stretching the sensors for 6000 times under cyclic loading at $50 \%$ strain showing the stability of the electrical signal response with less than $2 \%$ degradation. (e) the electrical signal frequency response of the VA-CNT-PDMS film at $50 \%$ strain from $0.25 \mathrm{~Hz}$ to up to $1 \mathrm{~Hz}$ with good identical response(f) Electrical hysteresis of the VA-CNT_PDMS sensor at different strain rates, with a small visible electrical hysteresis when stretched $60 \%$. 
The orientation of the CNTs in PDMS directly affects the sensing abilities and the mechanical response of the strain sensors. In literature, this directional sensing has not been considered although the different responses can assist the detection of multidirectional motions that the human body generates. For example, small-scale sensitive human motions, such as pulsing ${ }^{23}$ or breathing ${ }^{28}$ can generate a more reliable and distinguishable signal with a compression strain sensor than tension, since the \% compression with respect to the overall thickness of the sensor $\left(l_{1} / l\right.$, as described in Figure 3$)$ could be larger than the $\%$ stretching with respect to overall length of the film. Therefore, for practical applications, both should be considered. Figure 6 illustrates an application of the directionally-oriented CNT-PDMS strain sensors for a human motion detection. The two sensor types are attached to a wrist joint, (Figure 6 (a)), where the HA-CNT-PDMS strain sensor is placed on the top of the wrist joint and the VA-CNT-PDMS strain sensor is placed underneath. Both sensors have solid wire connections attached on top of the composite film via silver paste. When the wrist is bent towards the ground, the HA top sensor is subjected to a tensile strain along the CNT alignment axis, resulting in an increase in the electrical resistance signal of the film, whereas the bottom VA sensor is under compression with forces acting normal to the film, leading to an increase in conductivity as it is compressed, showing a negative $\left(\Delta R / R_{0}\right)$, as demonstrated in Figure 4a. Electrical signal measurements are performed simultaneously and then the sensor positions are swapped for comparison. 


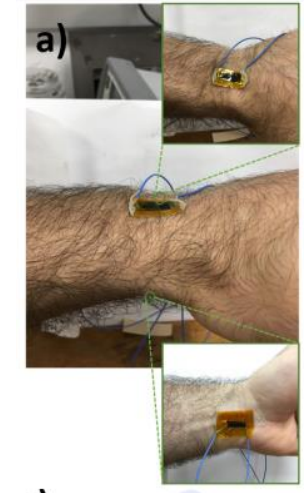

c)
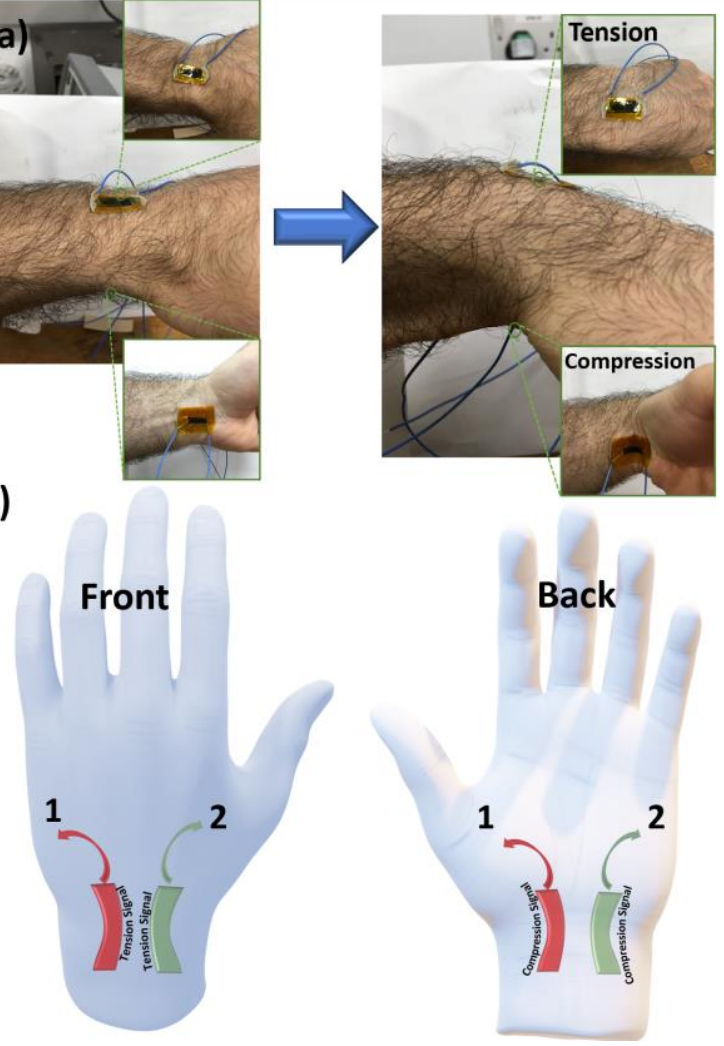
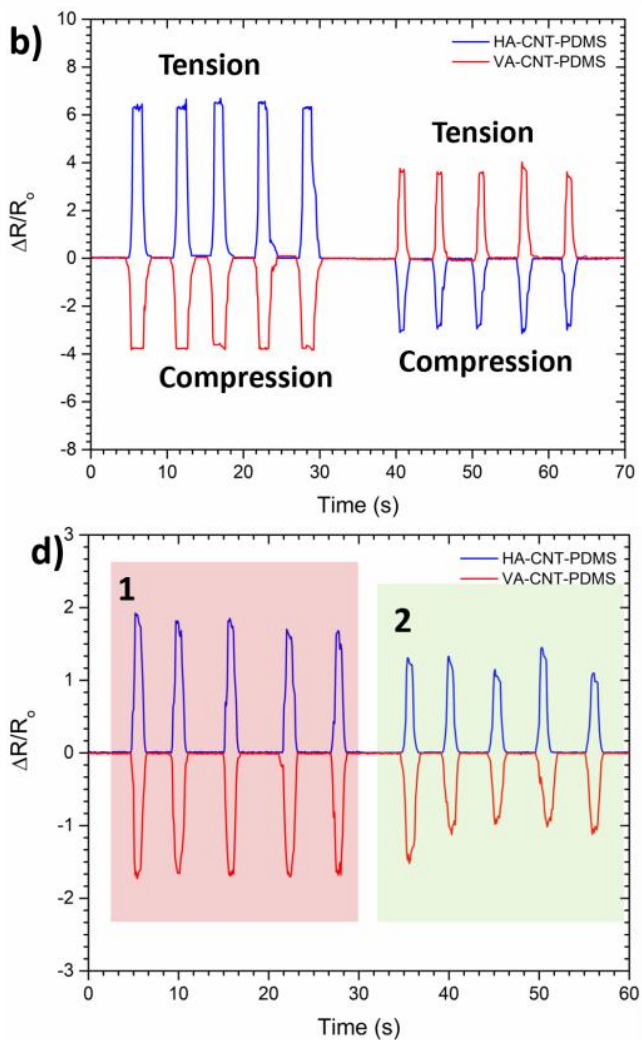

Figure 6. Application of the directionally oriented CNT-PDMS strain sensors to detect the wrist-joint movements multidirectionally. (a) is the initial resting position of the wrist, with HA-CNT-PDMS strain sensor on the top of the wrist and VACNT-PDMS strain sensor on the bottom of the wrist (inset, zoomed in image) and then the wrist in bending position indicated with the blue arrow, with the upper sensor in tension and the lower sensor in compression. (b) Electrical resistance changes of two types of sensors; VA-CNT-PDMS under tension (top of the wrist) and HA-CNT-PDMS under compression (bottom side of the wrist). After sensors are swapped, VA-CNT-PDMS under compression and HA (bottom side of the wrist) -CNT-PDMS under tension (top of the wrist) as described in Figure 4a. (c) A visualization of the multidirectional sensing testing, first the wrist is bent towards direction 1 and then in direction 2 (d) Electrical resistance changes when the wrist is bent left and right as visualised in (c), HACNT-PDMS sensor is placed on the front of the wrist, whereas VA-CNT-PDMS is placed on the back. The electrodes are placed on the tension side of and compression side of the VA-CNT-PDMS.

Figure $6 \mathrm{~b}$ shows the wrist-joint movement sensing performance of the CNT-PDMS strain sensors along the axis of a human hand. and record the corresponding relative electrical resistance changes $\left(\Delta R / R_{0}\right)$ subjected to repeating wrist bending. When the directional strain-sensing responses of the films are compared, we observe a few differences. Firstly, HA-CNTs produced a larger electrical signal $\left(\Delta R / R_{0}\right)$ under tension, however VA-CNTs perform better under compression. Secondly, under compression, VA-CNT sensors react to the movement slightly earlier than HA-CNT sensors, even though the difference is small; this could be the result of the difference in the electron transport mechanism explained in Figure 3 . To demonstrate multidirectional sensing abilities further, we test the sensors by subjecting them to side-to- 
side movement of the wrist in " 1 " and " 2 " direction as displayed in Figure 6c. HA-CNT-PDMS sensor is mounted on the front of the wrist, and the VA-CNT PDMS is mounted on the back. Then the electrical signals are collected from the tension side of the HA-CNT-PDMS and compression side of the VA-CNTPDSM sensors by placing the electrodes on corresponding sides respectively, shown in Figure 6c. It is possible to detect the movement not only along the axis of the hand but also across it (Figure $6 \mathrm{~d}$ ). Additionally, when moved in direction " 1 " the signal is stronger, allowing the opportunity to distinguish the movements. Similar to the results in Figure $6 \mathrm{~b}$, aligning the CNTs horizontally results in stronger electrical signal under tension, however vertically aligning the CNTs and collecting the signal from the compression side provides good electromechanical response. Wang et al. ${ }^{27}$ investigated a human's wrist movement using rosette-type printed CNT-PDMS strain sensors and argued that accurate movement monitoring could require multi-directional sensing. Other studies however, only focused on the singledirectional tensile strain for similar applications. ${ }^{23,30}$ Additionally, in these studies CNTs are deposited on to the substrate as a thin separate layer via printing ${ }^{27}$, spray coating ${ }^{30}$ or drop casting ${ }^{23}$ therefore they are not embedded in an elastomer, this raises the issue of physically damaging the thin film of CNTs as well as causing wrinkling and peeling effects due to the lack of a cover layer. ${ }^{24}$ Here, we show a simple method to fabricate embedded-CNT-PDMS nanocomposites with increased strength due to the alignment of the CNTs. Moreover, the process allows for the alignment of CNTs in certain directions providing extra sensitivity. Depending on the human body motion monitoring applications, directionally aligning the CNTs can improve the strain sensing abilities and help monitor more complicated multidirectional mechanical deformation induced strains. 

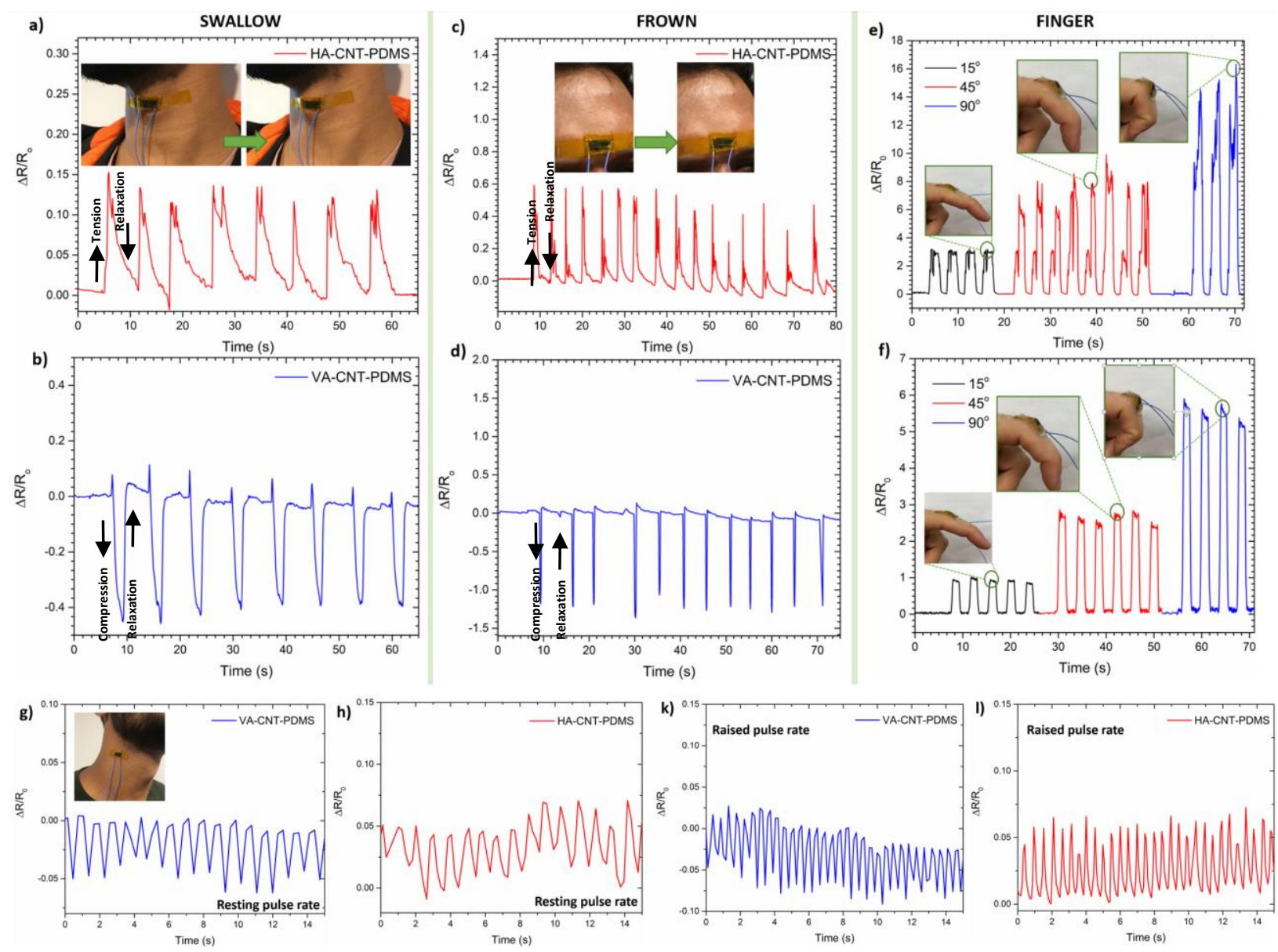

Figure 7. Sensitive strain sensing applications of the sensors for detecting; (a) plots the swallowing motion detection on the laryngeal prominence on the throat when data is collected via the tension side of the HA-CNT-PDMS strain sensor (b) swallowing motion detection on the laryngeal prominence on the throat when data is collected via the compression side of the VA-CNTPDMS strain sensor. (c) Detection of the "frowning" facial expression via the tension side of the HA-CNT-PDMS strain sensor. (d) Detection of the "frowning" facial expression via the compression side of the VA-CNT-PDMS strain sensor. (e) Monitoring of the motion of the index finger bending with three different ranges of motion with the HA-CNT-PDMS strain sensor (in tension). ( $f$ ) Monitoring of the motion of the index finger bending with three different ranges of motion with the VA-CNT-PDMS strain sensor (in tension). Real-time pulse monitoring over the carotid artery at resting heart rate (g) VA-CNT-PDMS and (h) HA-CNT-PDMS; at raised heart rate (k) VA-CNT-PDMS, (I) HA-CNT-PDMS.

Showing high directional sensitivity $(100>$ GF), combined with good stretchability/compressibility (50-

$55 \%)$ and mechanical robustness, these strain sensors have the potential to be employed to monitor various body motion induced strains. Not only limited to larger-scale human motion monitoring, we further test fabricated strain sensors for sensitive human motion detection applications, as plotted in Figure 7. Firstly, two types of strain sensors are placed on the laryngeal prominence on a male human's throat to monitor the swallowing motion (Figure $7(a$ and $b)$ ). In this test, the change in the electrical resistance is measured from the tension side of the HA-CNT-PDMS strain sensor by connecting the wires 
with silver paste on the same side. For measurements performed with the VA-CNT-PDMS strain sensors, wires are placed on the compression side of the film (Figure 7b). Figure 7a shows the generated electrical signal of the swallowing motion measured with HA-CNT-PDMS attached on to the laryngeal prominence, showing the tension and the relaxation of the muscle. VA-CNT-PDMS sensors provided a larger electrical signal $\left(\Delta R / R_{0}\right)$ for the same motion (Figure $\left.7 b\right)$. Although the signal is larger, VA-CNT sensors exhibit small overshoots as a result of the changes in the applied strains opposite to the stress direction, most probably due to the viscoelasticity of the elastomer combined with the stress relaxation effect. ${ }^{30,67}$ However, the VA-CNT sensors recovery time to the initial steady-state is on average $\sim 2 \mathrm{~s}$, compared to the HA-CNT films' average $\sim 5 \mathrm{~s}$. This could be explained by how the load is transferred under tensile and compressive stresses. Under mechanical deformation, the forces progress in the PDMS matrix and then are transferred to CNTs resulting in rearrangements and reorientations of the CNTs within the elastomer matrix. It is possible that the stress relaxation effect is more dominant in the tensile direction compared to compression resulting in the signal taking longer to reach steady state causing a drift. Given that the tested CNT-PDMS film thicknesses are much smaller than the length of the sensors, the distance required for the films under compression to relax back to their original state is smaller than films subjected to tension strains. Lee et al. ${ }^{30}$ argued that relaxation effect caused a reduction in the tensile strength leading to the electrical signal to drift more when it reached back to steady-state. They observed this kind of behaviour with the more sensitive, low density CNT sprayed PDMS films and showed that this was closely related with the sensitivity and stability of the sensors. Comparable results are reported here. ${ }^{22}$ We show a similar behaviour for the HA-CNT-PDMS sensors, however this explains the behaviour of more sensitive VA-CNTPDMS electrical signal shown in Figure 7b.

We further test the strain sensors to detect a frowning motion on the forehead as displayed in Figure 7(c,d). Both films provided good electrical signals, VA-CNTs showing excellent stability with a small overshoot whereas HA-CNTs showing a smaller signal with a small drift even though the frequency of this 
motion is larger. Firstly, the recovery time was below $\sim 0.5 \mathrm{~s}$ for VA-CNT sensors, and $\sim 3 \mathrm{~s}$ for the HA-CNTs. These values are smaller than Yamada et al.' $s^{5}$ CNT thin film-elastomer ( $\sim 5$ ) and Mattmann et al.'s $s^{68}$ carbon black fiber-elastomer sensors (100 s). A $90 \%$ time constant $\left(\tau_{90 \%}\right)$ is generally accepted as the standard response time value for stretchable sensors. ${ }^{24}$ The best response time observed are $\sim 160 \mathrm{~ms}$ and 130 ms for HA-CNT-PDMS and VA-CNT-PDMS sensors, respectively. These response times are considerably smaller than other CNT based stretchable sensors, for instance CNT-Ecoflex nanocomposite strain sensors are reported to have 332 ms response time. ${ }^{7}$ This could be the result of the very soft Ecoflex polymer, providing less driving force for a rapid restoration of the CNT network compared to PDMS with higher interfacial bonding. ${ }^{69}$ The sensitivities of the strain sensors are additionally tested by attaching the sensors on to a human index finger. In this test, the relative resistance changes of the films are recorded from the tension side by subjecting them to repeating finger bending, with results displayed in Figure 7(e and f). $\triangle R / R_{0}$ of the HA-CNT-PDMS (Figure 7e) and VA-CNT-PDMS sensors (Figure $7 f$ ) both increased when subjected to increased bending angles of $15^{\circ}, 45^{\circ}$ and $90^{\circ}$ respectively, allowing for easy discrimination. It should be noted here that, when used under tension, VA-CNT sensors show a smaller gain compared to HA-CNTs. This supports the findings discussed in Figure $6(k, l)$ and also Figure $7(a-d)$, aligning the CNTs in certain direction and utilising the CNT-PDMS sensors as tension or compression sensors depending on the sensing application can improve the sensing ability of the CNT-elastomer strain sensors.

Not limited to the human motion surveillance, sensors are utilized to monitor the pulse rate of a male subject by placing the sensors over the carotid artery both at resting heart rates Figure $7(\mathrm{~g}, \mathrm{~h})$ and raised heart rates Figure $7(\mathrm{k}, \mathrm{l})$. Figure $7 \mathrm{~g}$ and $\mathrm{h}$ show the carotid arterial pressure waveforms measured by VACNT-PDMS and HA-CNT-PDMS sensors, respectively. Both sensors calculate the pulse rate as 68 times per minutes, measured back to back. Additionally, the subject's pulse rate has been increased and measurements are repeated again with both sensors showing the increased heart rates with the VA-CNTPDMS sensor calculating 128 times per minute (Figure 7k) and the HA-CNT-PDMS sensor 120 times per 
minute (Figure 7l). The difference in the pulse rates measured is simply because of the difference in the intensity of the physical exercise the subject is exposed to as well as the variations of the resting time before the measurement.

The muscle/joint motions the human body generate are usually multi-directional and can require an array of sensor responses for more accurate measurements, we believe that distinguishable responses of the sensors tested combined with the improved electrical resistance signal sensitivity by aligning the CNTs in certain directions, can enable precise, small-scale human motion detection analysis and human-machine interface technology.

\section{Conclusion:}

In summary, we developed a very simple, low cost, high-yield technique to fabricate highly-sensitive and highly-stretchable directionally-aligned CNT-based strain sensors. Rolling CNTs horizontally on the substrate and then embedding them in to PDMS elastomer provided CNT alignment along the stretching direction as well as scale-like structures within the composite film limiting the conduction pathways which results in improved sensitivity at high tensile strain compared to vertically aligned CNT-PDMS strain sensors. At $50 \%$ tensile strain, GF values of 65 for HA-CNT-PDMS and 52 for VA-CNT-PDMS sensors are achieved. Sensors showed more sensitivity at smaller strains, 594 at 15\% for HA-CNT-PDMS and 326 at 25 $\%$ for the VA-CNT-PDMS, respectively. At $55 \%$ compressive strain, absolute GFs values reached 3.2 and 2.7 for VA-CNT-PDMS and HA-CNT-PDMS sensors respectively, similarly higher sensitivity ( 20 GF) at smaller strains (up to $25 \%$ compressive strains). Additionally, we observe that the CNT-PDMS strain sensors' electrical resistance response signal is different under tensile and compressive strains. Under small strains $(<10 \%)$, employing VA-CNT sensors under compression can help improve the signal compared to monitoring a similar mechanical deformation via a HA-CNT sensor under tension. Fabricated CNT-PDMS sensors are tested for over 6000 cycles under tension and compression at $50 \%$ strains and maintained their mechanical robustness as well as electromechanical performance. Response and 
recovery times as small as $130 \mathrm{~ms}$ and $0.5 \mathrm{~s} ; 160 \mathrm{~ms}$ and $2 \mathrm{~s}$ are reported for VA-CNT-PDMS and HA-CNTPDMS sensors, respectively. Both sensors can detect human motion induced mechanical deformations multidirectionally, both large and small-scale such as; pulse monitoring, swallowing, frowning, wrist and finger bending with ability to distinguish the motions. Additionally, this could help people with severe disabilities and limited movement to translate small muscle movements into electrical signals that can be developed into gestures/new alphabet to help communication.

\section{Supporting Information:}

Comparison graph for PDMS based stretchable strain sensors (S1), visualisation of electromechanical stretching testing setup (S2), surface roughness analysis of the substrate (S3), average CNT-PDMS film resistance measured as a function of applied compressive strain \% (S4), cross-section SEM images of the VA-CNT-PDMS sensor under different uniaxial tensile strains (S5), top view SEM images of the HA-CNTPDMS sensor under different uniaxial tensile strains (S6), detailed SEM images of the HA-CNT-PDMS and VA-CNT-PDMS sensors showing important sensing features (S7), gauge factor calculation formula.

\section{Notes:}

The authors declare no competing financial interest.

\section{Acknowledgements:}

The authors are grateful to Smiths Interconnect for the studentship funding and to EPSRC grant funding EP/N006372/1. The authors would also like to offer special thanks to Dr Vimal Gopee and National Physical Laboratory for allowing us to borrow certain components of the electromechanical testing setup. Finally, Mr Ash Stott's help with the human motion sensing measurements is greatly acknowledged. 


\section{Table of Contents Graphic:}

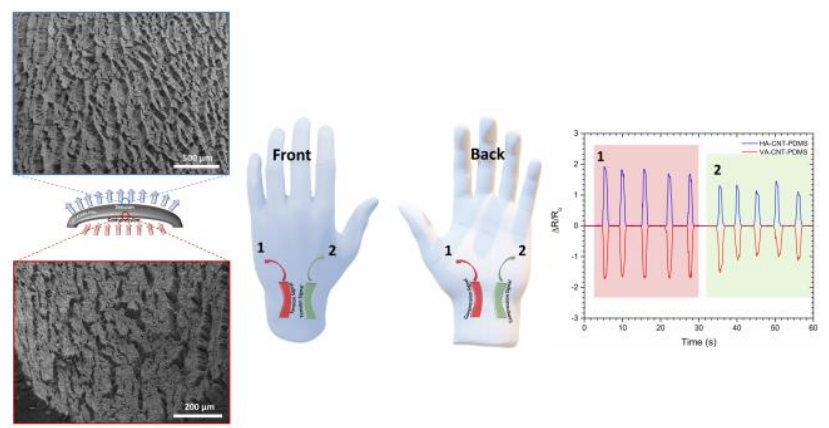

\section{References:}

(1) Park, S.; Vosguerichian, M.; Bao, Z. A Review of Fabrication and Applications of Carbon Nanotube Film-Based Flexible Electronics. Nanoscale 2013, 5 (5), 1727-1752. https://doi.org/10.1039/c3nr33560g.

(2) Son, D.; Lee, J.; Qiao, S.; Ghaffari, R.; Kim, J.; Lee, J. E.; Song, C.; Kim, S. J.; Lee, D. J.; Jun, S. W. Multifunctional Wearable Devices for Diagnosis and Therapy of Movement Disorders. Nat. Nanotechnol. 2014, 9 (5), 397-404. https://doi.org/10.1038/nnano.2014.38.

(3) Chortos, A.; Bao, Z. Skin-Inspired Electronic Devices. Mater. Today 2014, 17 (7), 321-331. https://doi.org/10.1016/j.mattod.2014.05.006.

(4) Amjadi, M.; Kyung, K. U.; Park, I.; Sitti, M. Stretchable, Skin-Mountable, and Wearable Strain Sensors and Their Potential Applications: A Review. Adv. Funct. Mater. 2016, 26 (11), 1678-1698. https://doi.org/10.1002/adfm.201504755.

(5) Yamada, T.; Hayamizu, Y.; Yamamoto, Y.; Yomogida, Y.; Izadi-Najafabadi, A.; Futaba, D. N.; Hata, K. A Stretchable Carbon Nanotube Strain Sensor for Human-Motion Detection. Nat. Nanotechnol. 2011, 6 (5), 296-301. https://doi.org/10.1038/nnano.2011.36.

(6) Chortos, A.; Liu, J.; Bao, Z. Pursuing Prosthetic Electronic Skin. Nat. Mater. 2016, 15 (9), 937-950. https://doi.org/10.1038/nmat4671.

(7) Amjadi, M.; Yoon, Y. J.; Park, I. Ultra-Stretchable and Skin-Mountable Strain Sensors Using Carbon Nanotubes-Ecoflex Nanocomposites. Nanotechnology 2015, 26 (37), 375501. https://doi.org/10.1088/0957-4484/26/37/375501.

(8) Huang, M.; Pascal, T. A.; Kim, H.; Goddard, W. A.; Greer, J. R. Electronic-Mechanical Coupling in Graphene from in Situ Nanoindentation Experiments and Multiscale Atomistic Simulations. Nano Lett. 2011, 11 (3), 1241-1246. https://doi.org/10.1021/nl104227t.

(9) Bae, S. H.; Lee, Y.; Sharma, B. K.; Lee, H. J.; Kim, J. H.; Ahn, J. H. Graphene-Based Transparent Strain Sensor. Carbon N. Y. 2013, 51 (1), 236-242. https://doi.org/10.1016/j.carbon.2012.08.048. 
(10) Hempel, M.; Nezich, D.; Kong, J.; Hofmann, M. A Novel Class of Strain Gauges Based on Layered Percolative Films of 2D Materials. Nano Lett. 2012, 12 (11), 5714-5718.

https://doi.org/10.1021/nl302959a.

(11) Yan, C.; Wang, J.; Kang, W.; Cui, M.; Wang, X.; Foo, C. Y.; Chee, K. J.; Lee, P. S. Highly Stretchable Piezoresistive Graphene-Nanocellulose Nanopaper for Strain Sensors. Adv. Mater. 2014, 26 (13), 2022-2027. https://doi.org/10.1002/adma.201304742.

(12) Herrmann, J.; Müller, K. H.; Reda, T.; Baxter, G. R.; Raguse, B.; De Groot, G. J. J. B.; Chai, R.; Roberts, M.; Wieczorek, L. Nanoparticle Films as Sensitive Strain Gauges. Appl. Phys. Lett. 2007, 91 (18), 89-92. https://doi.org/10.1063/1.2805026.

(13) Yao, S.; Zhu, Y. Wearable Multifunctional Sensors Using Printed Stretchable Conductors Made of Silver Nanowires. Nanoscale 2014, 6 (4), 2345-2352. https://doi.org/10.1039/c3nr05496a.

(14) Xiao, X.; Yuan, L.; Zhong, J.; Ding, T.; Liu, Y.; Cai, Z.; Rong, Y.; Han, H.; Zhou, J.; Wang, Z. L. HighStrain Sensors Based on ZnO Nanowire/Polystyrene Hybridized Flexible Films. Adv. Mater. 2011, 23 (45), 5440-5444. https://doi.org/10.1002/adma.201103406.

(15) Hu, L.; Kim, H. S.; Lee, J. Y.; Peumans, P.; Cui, Y. Scalable Coating and Properties of Transparent, Flexible, Silver Nanowire Electrodes. ACS Nano 2010, 4 (5), 2955-2963.

https://doi.org/10.1021/nn1005232.

(16) De, S.; Higgins, T. M.; Lyons, P. E.; Doherty, E. M.; Nirmalraj, P. N.; Blau, W. J.; Boland, J. J.; Coleman, J. N. Silver Nanowire Networks as Flexible, Transparent, Conducting Films: Extremely High DC to Optical Conductivity Ratios. ACS Nano 2009, 3 (7), 1767-1774.

(17) Gao, Q.; Li, H.; Zhang, J.; Xie, Z.; Zhang, J.; Wang, L. Microchannel Structural Design For a RoomTemperature Liquid Metal Based Super-Stretchable Sensor. Sci. Rep. 2019, 9 (1), 1-8. https://doi.org/10.1038/s41598-019-42457-7.

(18) Choi, D. Y.; Kim, M. H.; Oh, Y. S.; Jung, S. H.; Jung, J. H.; Sung, H. J.; Lee, H. W.; Lee, H. M. Highly Stretchable, Hysteresis-Free Ionic Liquid-Based Strain Sensor for Precise Human Motion Monitoring. ACS Appl. Mater. Interfaces 2017, 9 (2), 1770-1780. https://doi.org/10.1021/acsami.6b12415.

(19) De Volder, M.; H., Tawfick. S.; Baughman, R.; Hart, J. Carbon Nanotubes: Present and Future Commercial Applications. Science. 2013, 339 (February), 535-539.

(20) Frank, Stefan, Philippe Poncharal, Z. L. Wang, W. A. de H. Carbon Nanotube Quantum Resistors. Science (80-. ). 1998, 280 (5370), 1744-1746. https://doi.org/10.1126/science.280.5370.1744.

(21) Hu, N.; Karube, Y.; Arai, M.; Watanabe, T.; Yan, C.; Li, Y.; Liu, Y.; Fukunaga, H. Investigation on Sensitivity of a Polymer/Carbon Nanotube Composite Strain Sensor. Carbon N. Y. 2010, 48 (3), 680-687. https://doi.org/10.1016/j.carbon.2009.10.012.

(22) Zhou, J.; Yu, H.; Xu, X.; Han, F.; Lubineau, G. Ultrasensitive, Stretchable Strain Sensors Based on Fragmented Carbon Nanotube Papers. ACS Appl. Mater. Interfaces 2017, 9 (5), 4835-4842. https://doi.org/10.1021/acsami.6b15195.

(23) Chen, S.; Wu, R.; Li, P.; Li, Q.; Gao, Y.; Qian, B.; Xuan, F. Acid-Interface Engineering of Carbon Nanotube/Elastomers with Enhanced Sensitivity for Stretchable Strain Sensors. ACS Appl. Mater. Interfaces 2018, 10 (43), 37760-37766. https://doi.org/10.1021/acsami.8b16591. 
(24) Amjadi, M.; Pichitpajongkit, A.; Lee, S.; Ryu, S.; Park, I. Highly Stretchable and Sensitive Strain Sensor Based on Silver Nanowire-Elastomer Nanocomposite. ACS Nano 2014, 8 (5), 5154-5163. https://doi.org/10.1021/nn501204t.

(25) Cohen, D. J.; Mitra, D.; Peterson, K.; Maharbiz, M. M. A Highly Elastic, Capacitive Strain Gauge Based on Percolating Nanotube Networks. Nano Lett. 2012, 12 (4), 1821-1825. https://doi.org/10.1021/nl204052z.

(26) Nebel, C. E. Electronic Properties of CVD Diamond. Semicond. Sci. Technol. 2003, 18 (3), S1-S11. https://doi.org/10.1088/0268-1242/18/3/301.

(27) Wang, X.; Li, J.; Song, H.; Huang, H.; Gou, J. Highly Stretchable and Wearable Strain Sensor Based on Printable Carbon Nanotube Layers/Polydimethylsiloxane Composites with Adjustable Sensitivity. ACS Appl. Mater. Interfaces 2018, 10 (8), 7371-7380. https://doi.org/10.1021/acsami.7b17766.

(28) Cai, L.; Song, L.; Luan, P.; Zhang, Q.; Zhang, N.; Gao, Q.; Zhao, D.; Zhang, X.; Tu, M.; Yang, F. Super-Stretchable, Transparent Carbon Nanotube-Based Capacitive Strain Sensors for Human Motion Detection. Sci. Rep. 2013, 3, 1-9. https://doi.org/10.1038/srep03048.

(29) Lipomi, D. J.; Vosgueritchian, M.; Tee, B. C. K.; Hellstrom, S. L.; Lee, J. A.; Fox, C. H.; Bao, Z. Skinlike Pressure and Strain Sensors Based on Transparent Elastic Films of Carbon Nanotubes. Nat. Nanotechnol. 2011, 6 (12), 788-792. https://doi.org/10.1038/nnano.2011.184.

(30) Lee, J.; Lim, M.; Yoon, J.; Kim, M. S.; Choi, B.; Kim, D. M.; Kim, D. H.; Park, I.; Choi, S. J. Transparent, Flexible Strain Sensor Based on a Solution-Processed Carbon Nanotube Network. ACS Appl. Mater. Interfaces 2017, 9 (31), 26279-26285.

https://doi.org/10.1021/acsami.7b03184.

(31) Roh, E.; Hwang, B. U.; Kim, D.; Kim, B. Y.; Lee, N. E. Stretchable, Transparent, Ultrasensitive, and Patchable Strain Sensor for Human-Machine Interfaces Comprising a Nanohybrid of Carbon Nanotubes and Conductive Elastomers. ACS Nano 2015, 9 (6), 6252-6261. https://doi.org/10.1021/acsnano.5b01613.

(32) Song, Y.; Lee, J. I.; Pyo, S.; Eun, Y.; Choi, J.; Kim, J. A Highly Sensitive Flexible Strain Sensor Based on the Contact Resistance Change of Carbon Nanotube Bundles. Nanotechnology 2016, 27 (20), 1-6. https://doi.org/10.1088/0957-4484/27/20/205502.

(33) Liu, H.; Gao, J.; Huang, W.; Dai, K.; Zheng, G.; Liu, C.; Shen, C.; Yan, X.; Guo, J.; Guo, Z. Electrically Conductive Strain Sensing Polyurethane Nanocomposites with Synergistic Carbon Nanotubes and Graphene Bifillers. Nanoscale 2016, 8 (26), 12977-12989. https://doi.org/10.1039/c6nr02216b.

(34) Zeng, Y.; Ci, L.; Carey, B. J.; Vajtai, R.; Ajayan, P. M. Design and Reinforcement: Vertically Aligned Carbon Nanotube-Based Sandwich Composites. ACS Nano 2010, 4 (11), 6798-6804.

(35) Lee, J.; Pyo, S.; Kwon, D. S.; Jo, E.; Kim, W.; Kim, J. Ultrasensitive Strain Sensor Based on Separation of Overlapped Carbon Nanotubes. Small 2019, 15 (12), 1-7. https://doi.org/10.1002/smll.201805120.

(36) Hecht, D.; Hu, L.; Gruner, G. Conductivity Scaling with Bundle Length and Diameter in Single Walled Carbon Nanotube Networks. Appl. Phys. Lett. 2006, 89, 133112.

https://doi.org/10.1063/1.2356999. 
(37) Pu, J.-H.; Zha, X.-J.; Zhao, M.; Li, S.; Bao, R.-Y.; Liu, Z.-Y.; Xie, B.-H.; Yang, M.-B.; Guo, Z.; Yang, W. 2D End-to-End Carbon Nanotube Conductive Networks in Polymer Nanocomposites: A Conceptual Design to Dramatically Enhance the Sensitivities of Strain Sensors. Nanoscale 2018, 10 (5), 2191-2198. https://doi.org/10.1039/C7NR08077H.

(38) Lee, J. H.; Kim, J.; Liu, D.; Guo, F.; Shen, X.; Zheng, Q.; Jeon, S.; Kim, J. K. Highly Aligned, Anisotropic Carbon Nanofiber Films for Multidirectional Strain Sensors with Exceptional Selectivity. Adv. Funct. Mater. 2019, 1901623, 1-11. https://doi.org/10.1002/adfm.201901623.

(39) Ahmad, M.; Anguita, J. V.; Stolojan, V.; Corless, T.; Chen, J. S.; Carey, J. D.; Silva, S. R. P. High Quality Carbon Nanotubes on Conductive Substrates Grown at Low Temperatures. Adv. Funct. Mater. 2015, 25 (28), 4419-4429. https://doi.org/10.1002/adfm.201501214.

(40) Chen, J.-S.; Stolojan, V.; Silva, S. R. P. Towards Type-Selective Carbon Nanotube Growth at Low Substrate Temperature via Photo-Thermal Chemical Vapour Deposition. Carbon N. Y. 2015, 84, 409-418. https://doi.org/10.1016/j.carbon.2014.12.023.

(41) Ahmad, M.; Anguita, J. V.; Stolojan, V.; Carey, J. D.; Silva, S. R. P. Efficient Coupling of Optical Energy for Rapid Catalyzed Nanomaterial Growth: High-Quality Carbon Nanotube Synthesis at Low Substrate Temperatures. ACS Appl. Mater. Interfaces 2013, 5 (9), 3861-3866. https://doi.org/10.1021/am400542u.

(42) Tan, Y. Y.; Jayawardena, K. D. G. I.; Adikaari, A. A. D. T.; Tan, L. W.; Anguita, J. V.; Henley, S. J.; Stolojan, V.; Carey, J. D.; Silva, S. R. P. Photo-Thermal Chemical Vapor Deposition Growth of Graphene. Carbon N. Y. 2012, 50 (2), 668-673. https://doi.org/10.1016/j.carbon.2011.09.025.

(43) Tas, M. O.; Baker, M. A.; Musaramthota, V.; Uppal, H.; Masteghin, M. G.; Bentz, J.; Boxshall, K.; Stolojan, V. Carbon Nanotube Micro-Contactors on Ohmic Substrates for on-Chip Microelectromechanical Probing Applications at Wafer Level. Carbon N. Y. 2019, 150, 117-127. https://doi.org/10.1016/J.CARBON.2019.04.115.

(44) Khachadorian, S.; Papagelis, K.; Scheel, H.; Colli, A.; Ferrari, A. C.; Thomsen, C. High Pressure Raman Scattering of Silicon Nanowires. Nanotechnology 2011, 22 (19).

https://doi.org/10.1088/0957-4484/22/19/195707.

(45) Dresselhaus, M. S.; Jorio, A.; Hofmann, M.; Dresselhaus, G.; Saito, R. Perspectives on Carbon Nanotubes and Graphene Raman Spectroscopy. Nano Lett. 2010, 10 (3), 751-758.

https://doi.org/10.1021/n1904286r.

(46) Pimenta, M. A.; Dresselhaus, G.; Dresselhaus, M. S.; Canc, L. G. Studying Disorder in GraphiteBased Systems by Raman Spectroscopy W. Phys. Chem. Chem. Phys. 2007, 9, 1276-1291. https://doi.org/10.1039/b613962k.

(47) G Dresselhaus, M.S. Dresselhaus, Jorio, Ado, A.G. Souza Filho, R. S. Raman Spectroscopy on Isolated Single Wall Carbon Nanotubes. Carbon N. Y. 2002, 40, 2043-2061. https://doi.org/10.1016/S0008-6223(02)00066-0.

(48) Sato, K.; Saito, R.; Oyama, Y.; Jiang, J.; Canc, L. G.; Pimenta, M. A.; Jorio, A.; Dresselhaus, G.; Dresselhaus, M. S. D-Band Raman Intensity of Graphitic Materials as a Function of Laser Energy and Crystallite Size. 2006, 427, 117-121. https://doi.org/10.1016/j.cplett.2006.05.107.

(49) M.S. Dresselhausa, G. Dresselhaus, R. Saitoc, A. J. Raman Spectroscopy of Carbon Nanotubes. Phys. Rep. 2005, 1136 (2), 175-186. https://doi.org/10.1016/j.physrep.2004.10.006. 
(50) Dresselhaus, M. S.; Dresselhaus, G. Single Nanotube Raman Spectroscopy. Acc. Chem. Res. 2002, 35 (12), 1070-1078.

(51) Wood, J. R.; Zhao, Q.; Frogley, M. D.; Meurs, E. R.; Prins, A. D.; Peijs, T.; Dunstan, D. J.; Wagner, H. D. Carbon Nanotubes: From Molecular to Macroscopic Sensors. Phys. Rev. B - Condens. Matter Mater. Phys. 2000, 62 (11), 7571-7575. https://doi.org/10.1103/PhysRevB.62.7571.

(52) Kim, J. H.; Hwang, J. Y.; Hwang, H. R.; Kim, H. S.; Lee, J. H.; Seo, J. W.; Shin, U. S.; Lee, S. H. Simple and Cost-Effective Method of Highly Conductive and Elastic Carbon Nanotube/Polydimethylsiloxane Composite for Wearable Electronics. Sci. Rep. 2018, 8 (1), 1-11. https://doi.org/10.1038/s41598-017-18209-w.

(53) Tas, M. O.; Banerji, A.; Lou, M.; Lukitsch, M. J.; Alpas, A. T. Roles of Mirror-like Surface Finish and DLC Coated Piston Rings on Increasing Scuffing Resistance of Cast Iron Cylinder Liners. Wear 2017, 376-377, 1558-1569. https://doi.org/10.1016/j.wear.2017.01.110.

(54) Chen, H.; Miao, L.; Su, Z.; Song, Y.; Han, M.; Chen, X.; Cheng, X.; Chen, D.; Zhang, H. FingertipInspired Electronic Skin Based on Triboelectric Sliding Sensing and Porous Piezoresistive Pressure Detection. Nano Energy 2017, 40 (June), 65-72. https://doi.org/10.1016/j.nanoen.2017.08.001.

(55) Yu, M., Oleg Lourie, Mark J. Dyer, Katerina Moloni, Thomas F. Kelly, R. S. R. Strength and Breaking Mechanism of Multiwalled Carbon Nanotubes Under Tensile Load. Science (80-. ). 2000, 287 (5453), 637-640. https://doi.org/10.1126/science.287.5453.637.

(56) Xin, Y.; Zhou, J.; Xu, X.; Lubineau, G. Laser-Engraved Carbon Nanotube Paper for Instilling High Sensitivity, High Stretchability, and High Linearity in Strain Sensors. Nanoscale 2017, 9 (30), 10897-10905. https://doi.org/10.1039/c7nr01626c.

(57) Doshi, S. M.; Thostenson, E. T. Thin and Flexible Carbon Nanotube-Based Pressure Sensors with Ultrawide Sensing Range. ACS Sensors 2018, 3 (7), 1276-1282.

https://doi.org/10.1021/acssensors.8b00378.

(58) Yamada, T.; Saito, T.; Suzuki, M.; Wilhite, P.; Sun, X.; Akhavantafti, N.; Fabris, D.; Yang, C. Y. Tunneling between Carbon Nanofiber and Gold Electrodes. J. Appl. Phys. 2010, 107 (4). https://doi.org/10.1063/1.3295901.

(59) Gui, X.; Cao, A.; Wei, J.; Li, H.; Jia, Y.; Li, Z.; Fan, L.; Wang, K.; Zhu, H.; Wu, D. Soft, Highly Conductive Nanotube Sponges and Composites with Controlled Compressibility. ACS Nano 2010, 4 (4), 2320-2326. https://doi.org/10.1021/nn100114d.

(60) Liu, Z.; Bajwa, N.; Ci, L.; Lee, S. H.; Kar, S.; Ajayan, P. M.; Lu, J.-Q. Densification of Carbon Nanotube Bundles for Interconnect Application. 2007 IEEE Int. Interconnect Technol. Conf. 2007, 201-203. https://doi.org/10.1109/IITC.2007.382389.

(61) Rogers, J. A.; Someya, T.; Huang, Y.; Sorensen, A. E.; Lian, J.; Greer, J. R.; Valdevit, L.; Carter, W. B.; Ge, Q.; Jackson, J. A. Materials and Mechanics for Stretchable Electronics. Science (80-. ). 2010, 327 (5973), 1603-1607. https://doi.org/10.1126/science.1182383.

(62) Lee, H.; Seong, B.; Moon, H.; Byun, D. Directly Printed Stretchable Strain Sensor Based on Ring and Diamond Shaped Silver Nanowire Electrodes. RSC Adv. 2015, 5, 28379-28384. https://doi.org/10.1039/C5RA01519G.

(63) Lu, N.; Lu, C.; Yang, S.; Rogers, J. Highly Sensitive Skin-Mountable Strain Gauges Based Entirely on 
Elastomers. Adv. Funct. Mater. 2012, 22 (19), 4044-4050.

https://doi.org/10.1002/adfm.201200498.

(64) Liu, C. X.; Choi, J. W. Analyzing Resistance Response of Embedded PDMS and Carbon Nanotubes Composite under Tensile Strain. Microelectron. Eng. 2014, 117, 1-7.

https://doi.org/10.1016/j.mee.2013.11.013.

(65) Kong, K. T. S.; Mariatti, M.; Rashid, A. A.; Busfield, J. J. C. Enhanced Conductivity Behavior of Polydimethylsiloxane (PDMS) Hybrid Composites Containing Exfoliated Graphite Nanoplatelets and Carbon Nanotubes. Compos. Part B Eng. 2014, 58, 457-462.

https://doi.org/10.1016/j.compositesb.2013.10.039.

(66) Jeong, Y. R.; Park, H.; Jin, S. W.; Hong, S. Y.; Lee, S. S.; Ha, J. S. Highly Stretchable and Sensitive Strain Sensors Using Fragmentized Graphene Foam. Adv. Funct. Mater. 2015, 25 (27), 4228-4236. https://doi.org/10.1002/adfm.201501000.

(67) Deguchi, S.; Hotta, J.; Yokoyama, S.; Matsui, T. S. Viscoelastic and Optical Properties of Four Different PDMS Polymers. J. Micromechanics Microengineering 2015, 25 (9). https://doi.org/10.1088/0960-1317/25/9/097002.

(68) Mattmann, C.; Clemens, F.; Tröster, G. Sensor for Measuring Strain in Textile. Sensors 2008, 8 (6), 3719-3732. https://doi.org/10.3390/s8063719.

(69) Song, X.; Liu, S.; Gan, Z.; Lv, Q.; Cao, H.; Yan, H. Controllable Fabrication of Carbon NanotubePolymer Hybrid Thin Film for Strain Sensing. Microelectron. Eng. 2009, 86 (11), 2330-2333. https://doi.org/10.1016/j.mee.2009.04.012. 